\title{
QUASIHARMONIC FIELDS
}

\author{
Tadeusz IWANIEC ${ }^{\mathrm{a}}$, Carlo SBORDONE $^{\mathrm{b}}$ \\ ${ }^{a}$ Department of Mathematics, Syracuse University, Syracuse, NY 13210, USA \\ b Dipartimento di Matematica e Applicazioni "R. Caccioppoli" Università, \\ Via Cintia - 80126, Napoli, Italy
}

Received 20 April 2000

ABSTRACT. - To every solution of an elliptic PDE there corresponds a quasiharmonic field $\mathcal{F}=[B, E]-a$ pair of vector fields with $\operatorname{div} B=0$ and curl $E=0$ which are coupled by a distortion inequality. Quasiharmonic fields capture all the analytic spirit of quasiconformal mappings in the complex plane. Among the many desirable properties, we give dimension free and nearly optimal $L^{p}$-estimates for the gradient of the solutions to the divergence type elliptic PDEs with measurable coefficients. However, the core of the paper deals with quasiharmonic fields of unbounded distortion, which have far reaching applications to the non-uniformly elliptic PDEs. As far as we are aware this is the first time non-isotropic PDEs have been successfully treated. The right spaces for such equations are the Orlicz-Zygmund classes $L^{2} \log ^{\alpha} L$. Examples we give here indicate that one cannot go far beyond these classes.

(C) 2001 L'Association Publications de l'Institut Henri Poincaré. Published by Elsevier B.V. All rights reserved

RÉSUMÉ. - A chaque solution d'une équation elliptique correspond un champ quasiharmonique $\mathcal{F}=[B, E]$, un couple de champs de vecteurs qui vérifient $\operatorname{div} B=0$ et rot $E=0$ et qui sont couplés par une inégalité de distorsion. Les champs harmoniques capturent l'esprit analytique des applications quasiconformes dans le plan complexe. Dans cet article sont fournies des estimations $L^{p}$ concernant le gradient de solutions faibles d'une équation elliptique aux dérivées partielles, indépendantes de la dimension et presque optimales. Sont aussi considérés des champs quasiharmoniques avec distorsion non bornée, hypothèse non sans conséquences sur les équations non uniformément elliptiques. A notre connaissance c'est la première fois que sont obtenus des résultats significatifs pour des équations non-isotropiques. Les bons espaces pour de telles équations sont les espaces $L^{2} \log ^{\alpha} L$. Des exemples montrent qu'il est difficile de s'éloigner de cette classe.

(C) 2001 L'Association Publications de l'Institut Henri Poincaré. Published by Elsevier B.V. All rights reserved

\section{Introduction}

Quasiharmonic fields grew out of our study of the Leray-Lions equation

$$
\operatorname{div} A(x, \nabla u)=0 \quad \text { in } \Omega \subset \mathbb{R}^{n}
$$

using methods from quasiconformal geometry. In dimension 2 the relevance of this equation to quasiconformal mappings has been evident to researchers for more than 
fourty years, beginning with R. Caccioppoli [12], R. Finn and J. Serrin [30], L. Bers and L. Nirenberg [7], I.N. Vekua [74], B. Bojarski [9], etc. The definitions we shall introduce here stress these ideas. When the equation is linear

$$
\operatorname{div} A(x) \nabla u=0,
$$

then it is customary to state the ellipticity condition as the double inequality for the eigenvalues of the symmetric matrix $A(x) \in \mathbb{R}^{n \times n}$

$$
K^{-1}|\xi|^{2} \leqslant\langle A(x) \xi, \xi\rangle \leqslant K|\xi|^{2} .
$$

The factor $K$ here can be a constant or a measurable function on $\Omega$, that is, $K=K(x) \geqslant$ 1 . It is possible to express (1.3), equivalently, by using just one inequality

$$
|\xi|^{2}+|A(x) \xi|^{2} \leqslant\left(K+\frac{1}{K}\right)\langle A(x) \xi, \xi\rangle
$$

for almost every $x \in \Omega$ and all $\xi \in \mathbb{R}^{n}$. We will adopt this inequality to clarify the ellipticity bounds for the nonlinear equation (1.1), namely

$$
|\xi|^{2}+|A(x, \xi)|^{2} \leqslant\left(K+\frac{1}{K}\right)\langle A(x, \xi), \xi\rangle
$$

There are two vector fields associated with a solution of the equation (1.1). The first one, denoted by $E=\nabla u(x)$, is curl free while the second, $B=A(x, \nabla u)$, is divergence free. Now, any pair $\mathcal{F}=[B, E]$ of vector fields $B, E \in L_{l o c}^{1}\left(\Omega, \mathbb{R}^{n}\right)$ with $\operatorname{div} B=0$ and curl $E=0$ will be referred to as div-curl couple. To follow closely the ideas of quasiconformal mappings we introduce the norm and the Jacobian of $\mathcal{F}$ :

$$
|\mathcal{F}|^{2}=|B|^{2}+|E|^{2} \quad \text { and } \quad J(x, \mathcal{F})=\langle B, E\rangle .
$$

The term $K$-quasiharmonic field, of distortion $K=K(x) \geqslant 1$, pertains to those div-curl couples which satisfy

$$
|\mathcal{F}(x)|^{2} \leqslant \mathcal{K}(x) J(x, \mathcal{F}), \quad \mathcal{K}=K+K^{-1} .
$$

We call $K=K(x)$ the distortion function of $\mathcal{F}$. Needless to say, the natural domain for quasiharmonic fields is the space $L_{l o c}^{2}\left(\Omega, \mathbb{R}^{n} \times \mathbb{R}^{n}\right)$. However, the matters being discussed in this paper will also require other $L^{p}$-spaces and some Orlicz classes, such as

$$
L^{P}=L^{2} \log L \quad \text { and } \quad L^{Q}=L^{2} \log ^{-1} L .
$$

Continuing this theme from a perspective of the complex function theory, we introduce the \pm components of $\mathcal{F}$

$$
\mathcal{F}^{-}=\frac{1}{2}(E-B) \quad \text { and } \quad \mathcal{F}^{+}=\frac{1}{2}(E+B) .
$$


In many ways, these vector fields can be viewed as analogues of the Cauchy-Riemann partials $\partial f / \partial \bar{z}$ and $\overline{\partial f / \partial z}$ of a complex function $f=f(z)$. Recall that quasiconformal mappings in the planar domains are governed by the complex Beltrami equation

$$
\frac{\partial f}{\partial \bar{z}}=\mu(z) \frac{\partial f}{\partial z}, \quad \text { where }|\mu(z)| \leqslant \frac{K-1}{K+1}<1 .
$$

The following result ties quasiharmonic fields to this PDE.

THEOREM 1. - To each $K$-quasiharmonic field $\mathcal{F}$ in $\Omega$ there corresponds a measurable matrix-valued function $\mu: \Omega \rightarrow \mathbb{R}^{n \times n}$ of the form

$$
\mu(x)=\lambda(x)[\mathbf{I}-2 a \otimes a],
$$

where $a=a(x)$ is a unit vector field and

$$
\lambda(x)=\|\mu(x)\|=\sup _{|\xi|=1}|\mu(x) \xi| \leqslant \frac{K(x)-1}{K(x)+1}<1 .
$$

Furthemore, $\mathcal{F}$ solves the linear equation

$$
\mathcal{F}^{-}(x)=\mu(x) \mathcal{F}^{+}(x)
$$

Observe that $\mu(x)$ is a multiple of the orthogonal matrix $\mathbf{I}-2 a \otimes a$, a legitimate reason for calling (1.13) the Beltrami equation. We refer to $\mu$ as the distortion tensor, or the Beltrami matrix of $\mathcal{F}$. That $\mathcal{F}$ satisfies (1.13) for suitable choice of $\mu(x) \in \mathbb{R}^{n \times n}$ is almost a tautology. Nevertheless, it is advantageous to investigate quasiharmonic fields by viewing them as solutions to this system of PDEs with measurable coefficients (the entries of $\mu$ ). When there is no distortion, then $\mu(x) \equiv 0$, and in this case we are reduced to the generalized Cauchy-Riemann system for the coordinate functions $\left(f^{1}, \ldots, f^{n}\right)$ of the vector field $\mathcal{F}^{+}$,

$$
\frac{\partial f^{i}}{\partial x_{j}}=\frac{\partial f^{j}}{\partial x_{i}}, \quad \sum_{i=1}^{n} \frac{\partial f^{i}}{\partial x_{i}}=0 .
$$

Thus $\mathcal{F}^{+}$consists of conjugate harmonic functions while the minus component is zero. Quasiharmonic fields provide a suitable framework for better understanding and creation of new types of the second order elliptic PDEs. Every Beltrami system gives rise to an equation for the potential function of the curl free component. That is, for the function $u$ determined locally by $\nabla u=E$, we have a linear PDE of divergence form

$$
\begin{aligned}
& \operatorname{div} \mathcal{A}(x) \nabla u=0, \\
& K^{-1}(x)|\xi|^{2} \leqslant\langle\mathcal{A}(x) \xi, \xi\rangle \leqslant K(x)|\xi|^{2},
\end{aligned}
$$

where $K(x)$ is precisely the distortion of $\mathcal{F}$. It is a striking consequence of our consideration that every solution to the nonlinear Leray-Lions equation actually satisfies its own linear equation (1.14). While gaining linearity we compromise no ellipticity bounds. When the original equation (1.1) happens to be linear, then the coefficient matrix 
of the new equation enjoys rather special structure, namely

$$
\mathcal{A}(x)=\frac{\mathbf{I}-\mu(x)}{\mathbf{I}+\mu(x)}=\frac{1-\lambda(x)}{1+\lambda(x)} \mathbf{I}+\frac{4 \lambda(x)}{1-\lambda^{2}(x)} a \otimes a .
$$

It is interesting to note that

$$
\operatorname{det} \mathcal{A}(x)=\left[\frac{1-\lambda(x)}{1+\lambda(x)}\right]^{n-2}
$$

which equals 1 in two dimensions. Integral estimates for quasiharmonic fields lean heavily on properties of a singular integral operator

$$
\mathbf{S}: L^{p}\left(\mathbb{R}^{n}, \mathbb{R}^{n}\right) \longrightarrow L^{p}\left(\mathbb{R}^{n}, \mathbb{R}^{n}\right), \quad 1<p<\infty,
$$

which we refer to as Hilbert transform in $\mathbb{R}^{n}$. By the definition, $\mathbf{S}$ acts as identity on gradient fields and as minus identity on divergence free fields. We will also consider this operator in wider classes of vector functions, as the need will arise. Based on recent studies of Riesz transforms [42,6] we obtain a dimension free estimate of the $p$-norm of this operator. One inference of this estimate is the following dimension free improvement of Meyers' regularity result [55] for the divergence elliptic equations with measurable coefficients.

THEOREM 2. - Let a Hölder conjugate pair $q<2<p$ be given by

$$
q=\frac{14 K-12}{7 K-5} \quad \text { and } \quad p=\frac{14 K-12}{7 K-7}
$$

where $K \geqslant 1$ is a constant.

(a) Every $K$-quasiharmonic field in $L_{\text {loc }}^{q}\left(\Omega, \mathbb{R}^{n} \times \mathbb{R}^{n}\right)$ actually belongs to $L_{\text {loc }}^{p}\left(\Omega, \mathbb{R}^{n}\right.$ $\left.\times \mathbb{R}^{n}\right)$.

(b) All solutions to the Leray-Lions equation (1.1) of the Sobolev class $W_{l o c}^{1, q}(\Omega)$ are in the space $W_{\text {loc }}^{1, p}(\Omega)$.

One could probably extend the range of these exponents by only requiring that

$$
\frac{2 K}{K+1}<q<p<\frac{2 K}{K-1} .
$$

As yet, this has been proved only in dimension two [53] by using Astala's area distortion theorem [1,2,28], see also [4] for interesting results at the end-points. We shall refer to the numbers

$$
q_{K}=\frac{2 K}{K+1} \leqslant 2 \quad \text { and } \quad p_{K}=\frac{2 K}{K-1} \geqslant 2
$$

as the critical exponents for the $K$-quasiharmonic fields. In higher dimensions, finding the critical exponents remains one of the outstanding problems in elliptic PDEs with measurable coefficients. 
We can now discuss the Dirichlet problem

$$
\left\{\begin{array}{l}
\operatorname{div} A(x) \nabla u=\operatorname{div} F \\
u \in W_{0}^{1, r}(\Omega)
\end{array}\right.
$$

where $F$ is a given vector field in $L^{r}\left(\Omega, \mathbb{R}^{n}\right)$. In view of the above results the Sobolev exponent will be in the interval

$$
\frac{14 K-12}{7 K-5} \leqslant r \leqslant \frac{14 K-12}{7 K-7}, \quad K>1 .
$$

If the domain $\Omega$ is sufficiently regular (for instance a ball, cube or the entire space in $\mathbb{R}^{n}$ ), then the above problem admits unique solution. The solution verifies a uniform bound

$$
\|\nabla u\|_{r} \leqslant C(K)\|F\|_{r} .
$$

Estimates with exponents smaller than $\frac{n}{n-1}$ open the way to a study of the equation with measure in the right hand side

$$
\operatorname{div} A(x) \nabla u=v .
$$

Here, $v$ is a Radon (signed) measure of finite total variation on $\Omega$. When $\Omega$ is a bounded regular domain one may use Green's function to express $v$ as $\operatorname{div} F$, where $F$ belongs to all Lebesgue spaces $L^{s}\left(\Omega, \mathbb{R}^{n}\right)$ with $1 \leqslant s<\frac{n}{n-1}$. Standard estimates for the Newtonian potential will reveal that

$$
\sup _{0<\varepsilon \leqslant 1}\left[\varepsilon f_{\Omega}|F|^{\frac{n-\varepsilon-1}{n-1}}\right]^{\frac{n-1}{n-\varepsilon}} \leqslant c(n) f_{\Omega}|d \nu| .
$$

As always, $f_{\Omega}$ stands for the integral mean over $\Omega$. Because of (1.24) we say that $F$ lies in the grand Lebesgue space $L^{\frac{n}{n-1}}\left(\Omega, \mathbb{R}^{n}\right)$. This is a Banach space in which the supremum at (1.24) provides us with the norm of $F$. Parallel to this concept is the grand Sobolev space $W_{0}^{1, \frac{n}{n-1}}(\Omega)$. It is at this stage where our results which depend critically on the exponent $r$ first make their implications.

THEOREM 3. - Let $\Omega$ be a cube in $\mathbb{R}^{n}$ and suppose that the distortion function for the equation (1.23) is bounded by a constant $K$ smaller than $\frac{7 n-12}{n-2}$, that is

$$
1 \leqslant K(x) \leqslant K<\frac{7 n-12}{n-2} .
$$

Then Eq. (1.23) admits unique solution $u$ in the space $W_{0}^{1, \frac{n}{n-1}}(\Omega)$. This solution verifies a uniform bound

$$
\|\nabla u\|_{\left.\frac{n}{n-1}\right)} \leqslant c(n, K) f_{\Omega}|d v|
$$


On account of (1.19) one may conjecture that Theorem 3 still holds for

$$
1 \leqslant K<\frac{n}{n-2}
$$

and that this bound for $K$ is sharp.

Matters are quite different if the distortion function $K=K(x)$ is unbounded. It is more or less clear that without restrictions on $K(x)$ the quasiharmonic fields will not retain their higher integrability properties. While it is certainly not apparent at this point it will be necessary to assume that

$$
\mathrm{e}^{\gamma K} \in L^{1}(\Omega) \text { for some } \gamma>0 \text {. }
$$

In order to fully benefit from the estimates for quasiharmonic fields one needs to investigate them under minimal possible integrability hypotheses. In PDEs it is customary to assume that the solutions have finite energy, that is

$$
\mathcal{E}[u]=\int_{\Omega}\langle A(x, \nabla u), \nabla u\rangle<\infty .
$$

Associated with this integral is the so-called stored energy of the div-curl field

$$
\mathcal{E}[\mathcal{F}]=\int_{\Omega} J(x, \mathcal{F}) d x<\infty .
$$

As the distortion function is unbounded, we observe that finite energy of $\mathcal{F}$ neither requires nor implies its $L^{2}$-integrability. Nevertheless, the exponential integrability assumption on $K$ at (1.28) places $K$-quasiharmonic fields of finite energy in the Orlicz space $L^{2} \log ^{-1} L$. This follows from (1.7) by Young's inequality. For the converse, recent advances in the theory of the Jacobians $[60,17,43,34,59]$, etc. prove extremely helpful. It can be shown that the energy (stored on a compact set) of a quasiharmonic field of class $L^{2} \log ^{-1} L$, regardless of its distortion function, is always finite [43,65]. Even more, if $K$ is in the exponential class, then for every $m \geqslant 0$ we have

$$
\left\{\begin{array}{l}
|\mathcal{F}| \in L^{2}(\log \log L)^{m} \log ^{-1} L, \\
J(x, \mathcal{F}) \in L(\log \log L)^{m},
\end{array}\right.
$$

on compact subsets. We shall not pursue these methods further because in this way one cannot reach even the natural domain for $\mathcal{F}$, which is the space $L_{\text {loc }}^{2}\left(\Omega, \mathbb{R}^{n} \times \mathbb{R}^{n}\right)$.

Before jumping to any conclusion, we emphasize the need for the exponent $\gamma=\gamma(n)$ at (1.28) to be sufficiently large. How large this exponent ought to be will be determined by the forthcoming estimates. Although our estimates vary with the sections, at the end $\gamma=\gamma(n)$ will be fixed. With such $\gamma$, we prove not only that $\mathcal{F} \in L_{\text {loc }}^{2}\left(\Omega, \mathbb{R}^{n} \times \mathbb{R}^{n}\right)$, but also reach the Zygmund space $L^{2} \log L$.

THEOREM 4 (Integrability theorem). - If $\mathrm{e}^{\gamma K} \in L_{l o c}^{1}(\Omega)$ with sufficiently large $\gamma=$ $\gamma(n)$, then every $K$-quasiharmonic field of class $L^{2} \log ^{-1} L\left(\Omega, \mathbb{R}^{n} \times \mathbb{R}^{n}\right)$ is locally in $L^{2} \log L\left(\Omega, \mathbb{R}^{n} \times \mathbb{R}^{n}\right)$. 
Theorem 4 is in effect special case of the general fact that for every $\alpha>-1$ there is $\gamma=\gamma(n, \alpha)>0$ with the following properties

$$
\mathrm{e}^{\gamma K} \in L_{l o c}^{1}(\Omega) \text { implies }|\mathcal{F}| \in L^{2} \log ^{\alpha} L_{l o c}(\Omega),
$$

see the forthcoming paper [57].

In quest of the smallest such exponent $\gamma$, called the critical point for regularity, we are faced with a challenging conjecture.

CONJECTURE 1.1. - The critical point $\gamma=\gamma(n, \alpha)$ for which the implication (1.32) holds is independent of the dimension and equals:

$$
\gamma(\alpha)=1+\alpha .
$$

One may ask how Theorem 4 translates into estimates for Eq. (1.1). When trying to answer this question it is important to realize that the associated quasiharmonic field $\mathcal{F}=[A(x, \nabla u), \nabla u]$ belongs to $L^{2} \log ^{-1} L_{l o c}\left(\Omega, \mathbb{R}^{n} \times \mathbb{R}^{n}\right)$ if and only if the solution $u$ has locally finite energy. Our next result seems to be the first of its kind; both bounds on uniform ellipticity from below and from above are relaxed.

THEOREM 5 (Local regularity). - Every finite energy solution of the Leray-Lions equation (1.1) satisfies

$$
\begin{aligned}
& \|\nabla u\|_{L^{2} \log L\left(\Omega^{\prime}\right)}^{2}+\|A(x, \nabla u)\|_{L^{2} \log L\left(\Omega^{\prime}\right)}^{2} \\
& \leqslant C_{K}\|\langle A(x, \nabla u), \nabla u\rangle\|_{L \log ^{2} L\left(\Omega^{\prime}\right)} \\
& \quad \leqslant C_{K}\left(\Omega^{\prime}, \Omega\right) \int_{\Omega}\langle A(x, \nabla u), \nabla u\rangle d x
\end{aligned}
$$

for compact subsets $\Omega^{\prime} \subset \Omega$.

Let us stress explicitely that we are dealing with genuine anisotropic equations; the ratio of the largest eigenvalue to the smallest one is equal to $K^{2}(x)$ and, therefore, need not to be bounded. For results concerning isotropic equations we refer the reader to [29, 51,58]. Special nonisotropic equations were studied by [72,15]. As a note of warning, no higher integrability conclusion, even $L^{2}$-integrability of the gradient, can be drawn if the exponent $\gamma=\gamma(n)$ fails to be sufficiently large, see Section 6.

To make clear meaning of the Dirichlet problem

$$
\left\{\begin{array}{l}
\operatorname{div} A(x) \nabla u=\operatorname{div} A(x) F \\
u(x)=0 \quad \text { on } \partial \Omega
\end{array}\right.
$$

one needs right function spaces. Denote by $\mathcal{H}_{0}^{1, A}(\Omega)$ the Sobolev class of functions with finite energy $\mathcal{E}[u]=\int\langle A(x) \nabla u, \nabla u\rangle d x$, which vanish on $\partial \Omega$ in the sense of distributions. If $\langle A(x) F, F\rangle \in L^{1}(\Omega)$ the existence and uniqueness of such solutions follows by variational principles, and the energy estimate is straightforward

$$
\int_{\Omega}\langle A(x) \nabla u, \nabla u\rangle d x \leqslant \int_{\Omega}\langle A(x) F, F\rangle d x .
$$


However, under slightly stronger assumption that $K F \in L^{2}\left(\Omega, \mathbb{R}^{n}\right)$, we obtain the desired $L^{2}$-integrability of the gradient and more

$$
\|\nabla u\|_{2}+\|A \nabla u\|_{2} \leqslant C_{\Omega}(n, K)\|K F\|_{2} .
$$

Here, $\Omega$ stands for a cube in $\mathbb{R}^{n}$.

A device for obtaining integral estimates for quasiharmonic fields and the governing PDEs is the Beltrami operator

$$
\mathbf{I}-\mu \mathbf{S}: L^{\Phi}\left(\mathbb{R}^{n}, \mathbb{R}^{n}\right) \rightarrow L^{\Phi}\left(\mathbb{R}^{n}, \mathbb{R}^{n}\right)
$$

acting in suitable Orlicz spaces, where

$$
\|\mu(x)\| \leqslant \frac{K(x)-1}{K(x)+1} .
$$

The goal is to invert this operator. It is obvious that $\mathbf{I}-\mu \mathbf{S}$ is invertible in all Lebesgue spaces $L^{r}\left(\mathbb{R}^{n}, \mathbb{R}^{n}\right)$ for which $\|\mu\|_{\infty}\|\mathbf{S}\|_{r}<1$. Theorem 2 is an immediate consequence of this observation. If $K=K(x)$ is only in the exponential class, say $\mathrm{e}^{\gamma K} \in L_{l o c}^{1}\left(\mathbb{R}^{n}\right)$, the question whether $\mathbf{I}-\mu \mathbf{S}$ has inverse brings us to one more restriction of $K(x)$ near infinity. It will be required that

$$
\mathrm{e}^{\gamma K} \in L^{1}\left(\mathbb{R}^{n}\right)+L^{\infty}\left(\mathbb{R}^{n}\right)
$$

which is certainly the case if $K$ is bounded outside a compact set.

THEOREM 6 (Invertibility theorem). - There exists a bounded linear operator

$$
\Pi: L^{2} \log ^{\alpha}\left(\mathbb{R}^{n}, d \omega\right) \rightarrow L^{2} \log ^{\alpha}\left(\mathbb{R}^{n}, d x\right)
$$

such that

$$
\boldsymbol{\Pi} \circ(\mathbf{I}-\mu \mathbf{S})=(\mathbf{I}-\mu \mathbf{S}) \circ \boldsymbol{\Pi}=\mathbf{I}
$$

for all $\alpha=-1,0,1$.

Here we have used Orlicz classes of vector fields in $\mathbb{R}^{n}$, with respect to the Lebesgue measure $d x$ and the weighted measure $d \omega=K^{2}(x) d x$. Note that the Beltrami operator $\mathbf{I}-\mu \mathbf{S}$ acts boundedly in either space.

While two recently appeared preprints [39] and [3] touch on similar themes, our results here are new and undoubtedly interesting. There has been some earlier and related work on mappings with unbounded distortion in the plane by G. David [21], P. Tukia [73], T. Iwaniec and V. Šverák [47] and more recent one by M.A. Brakalova and J.A. Jenkins [10], V. Ryazanov, U. Srebro, and E. Yakubov [64]. In higher dimensions, we refer to J. Manfredi and E. Villamor [54], J. Heinonen and P. Koskela [37], and L. Migliaccio and G. Moscariello [56]. No attempt has been made in this paper to provide complete theory of the quasiharmonic fields. But at least one might notice that these fields are crucial for understanding the elliptic PDEs with measurable coefficients. 


\section{Part One:}

\section{Requisites from the Field Theory in $\mathbf{R}^{n}$}

Essential to our development is establishing notation, some technical details and an exposition of the vector fields in $\mathbf{R}^{n}$. The book by E. Stein [69] is particularly useful here.

\section{Div-curl couples}

Let $\Omega$ be a domain in $\mathbb{R}^{n}, n \geqslant 2$. We shall consider Schwartz distributions on $\Omega$ with values in $\mathbb{R}^{n}$, that is, $n$-tuples $F=\left(f^{1}, \ldots, f^{n}\right) \in \mathcal{D}^{\prime}\left(\Omega, \mathbb{R}^{n}\right)$ whose coordinate terms are in $\mathcal{D}^{\prime}(\Omega)$. It includes the Lebesgue space $L^{p}\left(\Omega, \mathbb{R}^{n}\right), 1 \leqslant p<\infty$, which we supply with the norm

$$
\|F\|_{p}=\left(\int_{\Omega}|F(x)|^{p} d x\right)^{1 / p} .
$$

Let $\mathbb{R}^{n \times n}$ denote the linear space of $n \times n$-matrices with real entries. As usual, for $X=\left[x_{i j}\right]$ and $Y=\left[y_{i j}\right]$ in $\mathbb{R}^{n \times n}$ the inner product is defined by $\langle X, Y\rangle=$ Trace $\left(X^{T} Y\right)=\Sigma x_{i j} y_{i j}$. If $F \in \mathcal{D}^{\prime}\left(\Omega, \mathbb{R}^{n}\right)$ we can speak of its differential

$$
D F=\left[\partial f^{i} / \partial x_{j}\right] \in \mathcal{D}^{\prime}\left(\Omega, \mathbb{R}^{n \times n}\right) .
$$

Then $F$ is said to be in the Sobolev class $\mathcal{W}^{1, p}\left(\Omega, \mathbb{R}^{n}\right)$ provided $D F \in L^{p}\left(\Omega, \mathbb{R}^{n \times n}\right)$. Let us emphasize explicitely that in this definition we do not require $F$ to be in $L^{p}\left(\Omega, \mathbb{R}^{n}\right)$. Modulo constant vector fields $\mathcal{W}^{1, p}\left(\Omega, \mathbb{R}^{n}\right)$ is a Banach space equipped with the seminorm

$$
\|F\|_{1, p}=\left(\int_{\Omega}|D F(x)|^{p} d x\right)^{1 / p} .
$$

The following two differential operators on the fields $F \in \mathcal{D}^{\prime}\left(\Omega, \mathbb{R}^{n}\right)$ will be of fundamental importance in this paper. The divergence $\operatorname{div}: \mathcal{D}^{\prime}\left(\Omega, \mathbb{R}^{n}\right) \rightarrow \mathcal{D}^{\prime}(\Omega, \mathbb{R})$ is a scalar distribution

$$
\operatorname{div} F=\frac{\partial f^{1}}{\partial x_{1}}+\cdots+\frac{\partial f^{n}}{\partial x_{n}}
$$

and the rotation $\operatorname{curl}: \mathcal{D}^{\prime}\left(\Omega, \mathbb{R}^{n}\right) \rightarrow \mathcal{D}^{\prime}\left(\Omega, \mathbb{R}^{n \times n}\right)$ is a matrix-valued distribution

$$
\operatorname{curl} F=\left[\frac{\partial f^{i}}{\partial x_{j}}-\frac{\partial f^{j}}{\partial x_{i}}\right] .
$$

Both operators owe much of their importance to the theory of Maxwell's equations and our notation reflects this relevance. For $F \in \mathcal{D}^{\prime}\left(\Omega, \mathbb{R}^{n}\right)$ and $\varphi \in C^{\infty}(\Omega)$ the following 
formulas will be useful

$$
\left\{\begin{array}{l}
D(\varphi F)=\varphi D F+F \otimes \nabla \varphi, \\
\operatorname{div}(\varphi F)=\varphi \operatorname{div} F+\langle F, \nabla \varphi\rangle, \\
\operatorname{curl}(\varphi F)=\varphi \operatorname{curl} F+F \star \nabla \varphi,
\end{array}\right.
$$

where the tensor product, inner product and curl product are defined by

$$
\begin{aligned}
& F \otimes \nabla \varphi=\left[f^{i} \frac{\partial \varphi}{\partial x_{j}}\right], \quad i, j=1, \ldots, n, \\
& \langle F, \nabla \varphi\rangle=\sum f^{i} \frac{\partial \varphi}{\partial x_{i}}=\operatorname{Trace}(F \otimes \nabla \varphi), \\
& F \star \nabla \varphi=\left[f^{i} \frac{\partial \varphi}{\partial x_{j}}-f^{j} \frac{\partial \varphi}{\partial x_{i}}\right]=F \otimes \nabla \varphi-\nabla \varphi \otimes F .
\end{aligned}
$$

Spaces of divergence free and curl free (irrotational fields) distributions will be denoted by using script letters

$$
\left\{\begin{array}{l}
\mathcal{B}(\Omega)=\left\{B \in \mathcal{D}^{\prime}\left(\Omega, \mathbb{R}^{n}\right) ; \operatorname{div} B=0\right\} \\
\mathcal{E}(\Omega)=\left\{E \in \mathcal{D}^{\prime}\left(\Omega, \mathbb{R}^{n}\right) ; \operatorname{curl} E=0\right\}
\end{array}\right.
$$

Note that the coordinates of a distribution $F=\left(f^{1}, \ldots, f^{n}\right)$ in the space $\mathcal{B}(\Omega) \cap \mathcal{E}(\Omega)$ satisfy the generalized Cauchy-Riemann system

$$
\left\{\begin{array}{l}
\frac{\partial f^{1}}{\partial x_{1}}+\cdots+\frac{\partial f^{n}}{\partial x_{n}}=0, \\
\frac{\partial f^{i}}{\partial x_{j}}=\frac{\partial f^{j}}{\partial x_{i}}, \quad i, j=1, \ldots, n .
\end{array}\right.
$$

Locally, such a field $F$ is the gradient of a harmonic function and, therefore, is a $C^{\infty}$ smooth vector field in $\Omega$. However, distributions which are only in $\mathcal{B}(\Omega)$ or $\mathcal{E}(\Omega)$ need not be represented by locally integrable functions. We denote by $\mathcal{B}^{p}(\Omega)$ and $\mathcal{E}^{p}(\Omega)$ the spaces of divergence free and curl free vector fields in $L^{p}\left(\Omega, \mathbb{R}^{n}\right)$, respectively. Of particular relevance to the boundary value problems is the space of gradient fields with vanishing tangential component

$$
\mathcal{E}_{0}^{p}(\Omega)=\left\{\nabla u ; u \in \mathcal{W}_{0}^{1, p}(\Omega)\right\} \subset \mathcal{E}^{p}(\Omega),
$$

where $\mathcal{W}_{0}^{1, p}(\Omega)$ stands for the completion of $C_{0}^{\infty}(\Omega)$ in $\mathcal{W}^{1, p}(\Omega)$. Now, the duality between div and curl can be stated as

$$
\int_{\Omega}\langle B(x), E(x)\rangle d x=0,
$$

whenever $E \in \mathcal{E}_{0}^{p}(\Omega)$ and $B \in \mathcal{B}^{q}(\Omega)$, where $1 \leqslant p, q \leqslant \infty$ is any Hölder conjugate pair. We skip discussing other possibilities for boundary constraints in (2.5) since a need 
will not arise. Let us point out, however, that $\mathcal{E}_{0}^{p}\left(\mathbb{R}^{n}\right)=\mathcal{E}^{p}\left(\mathbb{R}^{n}\right)$ and

$$
\int_{\mathbb{R}^{n}}\langle B(x), E(x)\rangle d x=0
$$

for $B \in \mathcal{B}^{q}\left(\mathbb{R}^{n}\right)$ and $E \in \mathcal{E}^{p}\left(\mathbb{R}^{n}\right), 1 / p+1 / q=1$. A central fact, hidden behind the forthcoming computation, is that the div-curl product $\langle B, E\rangle$ actually belongs to the Hardy space $\mathcal{H}^{1}\left(\mathbb{R}^{n}\right)$ [17]. This will be extended further to suitable pairs of conjugate Orlicz spaces.

DEFINITION 2.1. - A div-curl couple on $\Omega$ is a pair $\mathcal{F}=[B, E]$ with $B \in \mathcal{B}(\Omega)$ and $E \in \mathcal{E}(\Omega)$.

The case $B=E$ reduces to the Cauchy-Riemann equations (2.3), and because of this we refer to such couples as harmonic fields.

\section{Quasiharmonic fields}

If a div-curl couple $\mathcal{F}=[B, E]$ consists of locally integrable vector fields we can speak of its modulus

$$
|\mathcal{F}(x)|=\left(|B(x)|^{2}+|E(x)|^{2}\right)^{1 / 2}
$$

and the Jacobian

$$
J(x, \mathcal{F})=\langle B(x), E(x)\rangle .
$$

Clearly, $2 J(x, \mathcal{F}) \leqslant|\mathcal{F}(x)|^{2}$ and equality occurs only for harmonic fields.

Definition 3.1. $-A$ div-curl couple $\mathcal{F}=[B, E] \in \mathrm{七}_{\text {loc }}^{1}\left(\Omega, \mathbb{R}^{n} \times \mathbb{R}^{n}\right)$ is called $K$-quasiharmonic field, with the distortion $K=K(x) \geqslant 1$, if

$$
|\mathcal{F}(x)|^{2} \leqslant \mathcal{K}(x) J(x, \mathcal{F}), \quad \text { where } \mathcal{K}(x)=K(x)+K^{-1}(x) .
$$

In terms of the \pm components of $\mathcal{F}$ the distortion inequality reads as

$$
\left|\mathcal{F}^{-}(x)\right| \leqslant \frac{K(x)-1}{K(x)+1}\left|\mathcal{F}^{+}(x)\right|,
$$

where we recall that $2 \mathcal{F}^{ \pm}=E \pm B$, respectively. Hence, harmonic fields are precisely those with the vanishing minus component.

We are now in a position to prove Theorem 1. For this, we introduce the notation

$$
\lambda(x)=\frac{\left|\mathcal{F}^{-}(x)\right|}{\left|\mathcal{F}^{+}(x)\right|} \quad \text { if } \mathcal{F}^{+}(x) \neq 0
$$

and put $\lambda(x)=0$, otherwise. As the vectors $\mathcal{F}^{-}(x)$ and $\lambda(x) \mathcal{F}^{+}(x)$ have the same length, there exists an isometry which takes $\lambda \mathcal{F}^{+}$into $\mathcal{F}^{-}$. There are of course many such isometries. However, for reasons to be discussed later, it will be advantageous to 
choose the reflection about the hyperplane bisecting the angle between these vectors. More precisely, the reflection we have chosen is represented by the orthogonal matrix

$$
I-2 a \otimes a,
$$

where $a=a(x)$ is the unit normal vector to the hyperplane in question. The tensor product $a \otimes a \in \mathbb{R}^{n \times n}$ is a symmetric matrix defined by the rule $[a \otimes a] v=\langle a, v\rangle a$ for $v \in \mathbb{R}^{n}$. More explicitely, if $\mathcal{F}^{-}(x) \neq \lambda(x) \mathcal{F}^{+}(x)$, then we have

$$
a(x)=\frac{\mathcal{F}^{-}(x)-\lambda(x) \mathcal{F}^{+}(x)}{\left|\mathcal{F}^{-}(x)-\lambda(x) \mathcal{F}^{+}(x)\right|} .
$$

Otherwise, $a(x)$ will be any unit vector perpendicular to $\mathcal{F}^{+}(x)$. The latter ensures that $[a \otimes a] \mathcal{F}^{+}=0$. In either case, we obtain the Beltrami equation

$$
\mathcal{F}^{-}(x)=\mu(x) \mathcal{F}^{+}(x)
$$

with $\mu(x)=\lambda(x)[\mathbf{I}-2 a \otimes a]$, establishing Theorem 1 .

It is worth noting that the Beltrami matrix $\mu$ of this form is unique except for the points where $\mathcal{F}^{-}(x)=\lambda(x) \mathcal{F}^{+}(x)$. In dimension 2 the case $\mathcal{F}^{-}(x)=\lambda(x) \mathcal{F}^{+}(x)$ still leads to the uniqueness of $\mu(x)$.

Locally we can always write $\mathcal{F}=[B, \nabla u]$ with $B \in \mathcal{B}_{\text {loc }}^{1}$ and $u \in \mathcal{W}_{\text {loc }}^{1,1}$. The Beltrami equation yields

$$
\mathcal{A}(x) \nabla u=B(x)
$$

where the matrix $\mathcal{A}(x)$ takes the form

$$
\mathcal{A}(x)=(\mathbf{I}-\mu(x))(\mathbf{I}+\mu(x))^{-1}=\frac{1-\lambda(x)}{1+\lambda(x)} \mathbf{I}+\frac{4 \lambda(x)}{1-\lambda^{2}(x)} a \otimes a .
$$

It is a simple matter of multiplying matrices to verify this explicit expression for $\mathcal{A}(x)$. An elementary computation shows that

$$
\begin{aligned}
\operatorname{det} \mathcal{A}(x) & =\left(\frac{1-\lambda}{1+\lambda}\right)^{n} \operatorname{det}\left[\mathbf{I}+\frac{4 \lambda}{(1-\lambda)^{2}} a \otimes a\right] \\
& =\left(\frac{1-\lambda}{1+\lambda}\right)^{n}\left[1+\frac{4 \lambda}{(1-\lambda)^{2}}\right]=\left[\frac{1-\lambda(x)}{1+\lambda(x)}\right]^{n-2}
\end{aligned}
$$

establishing formula (1.16). that

The only point remaining concerns the ellipticity bounds. It is immediate from (3.8)

$$
\langle\mathcal{A}(x) \xi, \xi\rangle=\frac{1-\lambda(x)}{1+\lambda(x)}|\xi|^{2}+\frac{4 \lambda(x)}{1-\lambda^{2}(x)}\langle a(x), \xi\rangle^{2} .
$$

We thus get

$$
\left(\frac{1-\lambda}{1+\lambda}\right)|\xi|^{2} \leqslant\langle\mathcal{A}(x) \xi, \xi\rangle \leqslant\left(\frac{1-\lambda}{1+\lambda}+\frac{4 \lambda}{1-\lambda^{2}}\right)|\xi|^{2}
$$


From the above and by $\lambda(x) \leqslant(K(x)-1) /(K(x)+1)$, we conclude with the desired lower and upper bounds for the eigenvalues of $\mathcal{A}(x)$

$$
K^{-1}(x)|\xi|^{2} \leqslant\langle\mathcal{A}(x) \xi, \xi\rangle \leqslant K(x)|\xi|^{2} .
$$

Next we put on stage quasiconformal mappings in the complex plane $\mathbb{C}=\{z=x+$ $i y, x, y \in \mathbb{R}\}$ and link them with the concept of quasiharmonic fields in $\mathbb{R}^{2}$.

\section{Quasiconformal mappings}

One of the first things we wish to point out is the Hodge star operator

$$
\star=\left[\begin{array}{cc}
0 & 1 \\
-1 & 0
\end{array}\right]: \mathbb{R}^{2} \rightarrow \mathbb{R}^{2} .
$$

It is immediate that $\star \star=-\mathbf{I}$. The Hodge operator transforms curl-free fields into div-free fields. If $E=\nabla u=\left(u_{x}, u_{y}\right)$, then $\star E=\left(u_{y},-u_{x}\right)$ and hence $\operatorname{div}(\star E)=0$.

Locally, a pair $\mathcal{F}=[B, E]$ is a div-curl couple if and only if $E=\nabla u$ and $B=* \nabla v$. Assuming that $u, v \in W_{l o c}^{1, p}(\Omega)$, we obtain a complex function $f(z)=u(x, y)+i v(x, y)$ in the Sobolev class $W_{\text {loc }}^{1, p}(\Omega, \mathbb{C})$. Recall that $f$ is $K$-quasiregular, with $1 \leqslant K=K(z)<$ $\infty$, if and only if

$$
|D f(z)|^{2} \leqslant \mathcal{K}(z) J(z, f), \quad \mathcal{K}=K+K^{-1},
$$

where $J(z, f)=u_{x} v_{y}-u_{y} v_{x}=\langle B, E\rangle$ and

$$
D f(z)=\left[\begin{array}{cc}
u_{x} & u_{y} \\
v_{x} & v_{y}
\end{array}\right] .
$$

The Hilbert-Schmidt norm of the Jacobian matrix is being used here, that is, $|D f(z)|^{2}=$ Trace $\left[D^{T} f(z) D f(z)\right]=u_{x}^{2}+u_{y}^{2}+v_{x}^{2}+v_{y}^{2}$. Of particular relevance to quasiconformal geometry is a distortion tensor $G: \Omega \rightarrow \mathbb{R}^{2 \times 2}$, defined by

$$
G(z)=\frac{D^{T} f(z) D f(z)}{J(z, f)} .
$$

Here at some points $J(z, f)$ may vanish and, as a convention, we understand that $G(z)$ is the unit matrix in such cases. We then define

$$
\mathcal{A}(z)=G^{-1}(z)=\frac{1}{u_{x} v_{y}-u_{y} v_{x}}\left[\begin{array}{cc}
u_{y}^{2}+v_{y}^{2} & -u_{x} u_{y}-v_{x} v_{y} \\
-u_{x} u_{y}-v_{x} v_{y} & u_{x}^{2}+v_{x}^{2}
\end{array}\right]
$$

and notice that

$$
K^{-1}(z)|\xi|^{2} \leqslant\langle\mathcal{A}(z) \xi, \xi\rangle \leqslant K(z)|\xi|^{2} .
$$

We also find that $B$ and $E$ are coupled by the equation

$$
\mathcal{A}(z) E=B .
$$


The same equation holds for the dual couple $\mathcal{F}^{\prime}=\left[B^{\prime}, E^{\prime}\right]$, with $E^{\prime}=-\nabla v$ and $B^{\prime}=\star \nabla u$. This is due to the following identity

$$
\star \mathcal{A} \star=-\mathcal{A}^{-1} .
$$

In particular, the real and the imaginary part of $f$ satisfy the same second order PDE

$$
\operatorname{div} \mathcal{A}(z) \nabla u=0 \quad \text { and } \quad \operatorname{div} \mathcal{A}(z) \nabla v=0 .
$$

Observe that in two dimensions the Beltrami matrix $\mu=\lambda(\mathbf{I}-2 a \otimes a)$ takes the following special form $\left[\begin{array}{cc}\alpha & \beta \\ \beta & -\alpha\end{array}\right]$. Under the usual identification of points in $\mathbb{R}^{2}$ with the complex numbers, we see that the linear transformation $\mu: \mathbb{C} \rightarrow \mathbb{C}$ maps $\xi$ into $(\alpha+i \beta) \bar{\xi}$.

We can now identify the complex partials $f_{\bar{z}}=\frac{1}{2}\left(f_{x}+i f_{y}\right)$ and $f_{z}=\frac{1}{2}\left(f_{x}-i f_{y}\right)$ with the \pm components of $\mathcal{F}$ by the rules

$$
\left\{\begin{array}{l}
\mathcal{F}^{-}(z)=\frac{1}{2}(E-B)=f_{\bar{z}}, \\
\mathcal{F}^{+}(z)=\frac{1}{2}(E+B)=\overline{f_{z}} .
\end{array}\right.
$$

This leads us to the familiar complex Beltrami equation $f_{\bar{z}}=(\alpha+i \beta) f_{z}$.

\section{Radial stretching}

One particular $K$-quasiconformal mapping proves useful, as it is extremal for various problems in PDEs. This is the radial stretching

$$
f(z)=z|z|^{\alpha}, \quad \alpha=\frac{1}{K}-1 \leqslant 0 .
$$

An elementary computation shows that

$$
2 f_{z}=(2+\alpha)|z|^{\alpha} \text { and } 2 f_{\bar{z}}=\alpha|z|^{\alpha-2} z^{2}
$$

which brings us to the complex Beltrami equation:

$$
f_{\bar{z}}=\mu(z) f_{z} \quad \text { with } \mu(z)=\frac{1-K}{1+K} \frac{z}{\bar{z}} .
$$

The coefficient matrix in (4.8) takes the form

$$
\mathcal{A}(z)=\frac{1}{K} \mathbf{I}+\left(K-\frac{1}{K}\right) \frac{z \otimes z}{|z|^{2}},
$$

where we record the following ellipticity bounds

$$
\frac{1}{K}=\min _{|\xi|=1}\langle\mathcal{A}(z) \xi, \xi\rangle \leqslant \max _{|\xi|=1}\langle\mathcal{A}(z) \xi, \xi\rangle=K .
$$


The real and imaginary parts of $f$ are:

$$
u(x, y)=x\left(x^{2}+y^{2}\right)^{\frac{1-K}{2 K}} \quad \text { and } \quad v(x, y)=y\left(x^{2}+y^{2}\right)^{\frac{1-K}{2 K}} .
$$

These are none other than J. Serrin's [66] solutions to Eqs. (4.8). They belong to the Sobolev space $W_{\text {loc }}^{1, p}\left(\mathbb{R}^{2}\right)$ for all $1 \leqslant p<2 K /(K-1)$, but not for $p \geqslant 2 K /(K-1)$. The Beltrami equation (5.2) also holds for the function $1 / f(z)$, which gives us another set of solutions to Eqs. (4.8), namely

$$
u^{\prime}(x, y)=x\left(x^{2}+y^{2}\right)^{-\frac{K+1}{2 K}} \quad \text { and } \quad v^{\prime}(x, y)=y\left(x^{2}+y^{2}\right)^{-\frac{K+1}{2 K}} .
$$

Note that we have simply replaced $K$ by $-K$ in (5.4). However, these latter ones are only very weak solutions. They belong to $W_{\text {loc }}^{1, q}\left(\mathbb{R}^{2}\right)$ for all $1 \leqslant q<\frac{2 K}{K+1}$, but not for $q \geqslant \frac{2 K}{K+1}$. Finally, regarding $u, v, u^{\prime}, v^{\prime}$ and $\mathcal{A}$ as functions of $n$ variables we conclude that in any dimension the critical exponents $q_{K}$ and $p_{K}$, defined in (1.19), are sharp.

\section{The failure of higher integrability}

Consider more general radial stretching

$$
f(z)=z \chi(|z|) .
$$

Here we assume that the function $t \mapsto t \chi(t)$ is strictly increasing while $\chi(t)$ is decreasing. Elementary computation gives

$$
\begin{aligned}
& \left|f_{z}\right|+\left|f_{\bar{z}}\right|=\chi(|z|), \\
& \left|f_{z}\right|-\left|f_{\bar{z}}\right|=\chi(|z|)+|z| \chi^{\prime}(|z|) .
\end{aligned}
$$

Hence the distortion function takes the form

$$
K(z)=\frac{\left|f_{z}\right|+\left|f_{\bar{z}}\right|}{\left|f_{z}\right|-\left|f_{\bar{z}}\right|}=\frac{\chi(|z|)}{\chi(|z|)+|z| \chi^{\prime}(|z|)} .
$$

We apply this formula to

$$
\chi(|z|)=\frac{1}{|z| \log ^{\sigma} \frac{1}{|z|}} \quad \text { for }|z|<\mathrm{e}^{-\sigma}, \sigma>0 .
$$

Thus

$$
K(z)=\frac{1}{\sigma} \log \frac{1}{|z|}
$$

and

$$
|\mathcal{F}|=\frac{1}{|z|} \log ^{-\sigma} \frac{1}{|z|} \sqrt{1+\sigma^{2} \log ^{-2} \frac{1}{|z|}}
$$


in the open ball $\Omega=\left\{z ;|z|<\mathrm{e}^{-\sigma}\right\}$. We see at once that $\mathrm{e}^{\gamma K} \in L^{1}(\Omega)$ for all exponents $\gamma<2 \sigma$. On the other hand $|\mathcal{F}| \notin L^{2} \log ^{\alpha}(\Omega)$ if $2 \sigma \leqslant 1+\alpha$. In conclusion, for the implication at (1.32) it is necessary to assume that $\gamma \geqslant 1+\alpha$, whenever $\alpha>-1$, confront with Conjecture 1.1.

\section{Other examples}

There are more examples directly linked with the quasiharmonic fields

Example 7.1. - Let $f=\left(f^{1}, \ldots, f^{n}\right): \Omega \rightarrow \mathbb{R}^{n}$ be a mapping whose coordinates $f^{i}$ are in $W^{1, p_{i}}(\Omega), 1<p_{1}, \ldots, p_{n}<\infty, i=1,2, \ldots, n$. With $f$ we associate two vector fields

$$
E=\nabla f^{1} \quad \text { and } \quad B=\nabla f^{2} \times \cdots \times \nabla f^{n} .
$$

The latter stands for the cross product of $n-1$ gradient fields in $\mathbb{R}^{n}$. It is well known that

$$
\operatorname{div} B=0
$$

provided $\frac{1}{p_{2}}+\cdots+\frac{1}{p_{n}} \leqslant 1$. By the Laplace expansion formula we see that the dot product $\langle B, E\rangle$ equals the Jacobian determinant of $f$. That is, $\langle B, E\rangle=\operatorname{det}[D f(x)]=J(x, f)$. Now, the couple $\mathcal{F}=[B, E]$ is a $K$-quasiharmonic field if and only if

$$
\left|\nabla f^{1}\right|^{2}+\left|\nabla f^{2} \times \cdots \times \nabla f^{n}\right|^{2} \leqslant \mathcal{K} \operatorname{det}[D f] .
$$

This inequality provides a method for constructing elliptic equations and their particular solutions, without getting into a lengthy calculation of the second order distributional derivatives. For a treatment of more general constructions we refer the reader to [44].

Example 7.2. - Let $\Phi: \Omega \times \mathbb{R}^{n} \rightarrow \mathbb{R}$ be a Carathéodory function such that $\xi \in \mathbb{R}^{n} \rightarrow \Phi(x, \xi)$ is convex. Denote by $\Phi^{*}(x, \eta)=\sup \left\{\langle\eta, \xi\rangle-\Phi(x, \xi) ; \xi \in \mathbb{R}^{n}\right\}$ the Young conjugate of $\Phi(x,$.$) . Throughout this example we assume the quadratic growth$ and coercivity condition. This can be expressed by a single inequality

$$
|\xi|^{2}+|\eta|^{2} \leqslant\left(K+\frac{1}{K}\right)\left[\Phi(x, \xi)+\Phi^{*}(x, \eta)\right]
$$

where $K \geqslant 1$. Let $u \in W^{1,2}(\Omega)$ be a local minimizer of the variational integral

$$
I[v]=\int_{\Omega} \Phi(x, \nabla v) d x .
$$

Precisely we mean that $I[u]=\min \left\{I[v] ; v \in u+W_{0}^{1,2}(\Omega)\right\}$. Consider the solution $B \in \mathcal{B}^{2}(\Omega)$ of the dual problem in the sense of Ekeland-Temam [27]. That is, $\operatorname{div} B=0$ in $\Omega$ and

$$
\int_{\Omega}\left[\langle B, \nabla u\rangle-\Phi^{*}(x, B)\right]=\max \left\{\int_{\Omega}\left[\langle X, \nabla u\rangle-\Phi^{*}(x, X)\right] ; X \in \mathcal{B}^{2}(\Omega)\right\} .
$$


Then the extremality relations take the form

$$
\langle B, \nabla u\rangle=\Phi(x, \nabla u)+\Phi^{*}(x, B) \text { a.e. in } \Omega .
$$

Setting $E=\nabla u \in \mathcal{E}^{2}(\Omega)$ we obtain a $K$-quasiharmonic field $\mathcal{F}=[B, E]$ satisfying the distortion inequality

$$
|B|^{2}+|E|^{2} \leqslant\left(K+\frac{1}{K}\right)\langle B, E\rangle .
$$

\section{Hodge decomposition and Poincaré lemmas}

For our purpose it will be sufficient to review div-curl decomposition in the entire space $\Omega=\mathbb{R}^{n}$. In this case we can make explicit calculations by means of the Riesz transforms

$$
\mathbf{R}=\left(\mathbf{R}_{1}, \ldots, \mathbf{R}_{n}\right): L^{p}\left(\mathbb{R}^{n}\right) \rightarrow L^{p}\left(\mathbb{R}^{n}, \mathbb{R}^{n}\right), \quad 1<p<\infty,
$$

where

$$
(\mathbf{R} f)(x)=\frac{\Gamma\left(\frac{n+1}{2}\right)}{\pi^{\frac{n+1}{2}}} \int_{\mathbb{R}^{n}} \frac{(x-y) f(y) d y}{|x-y|^{n+1}} .
$$

The book by E. Stein [69] is a particularly good reference. Consider a vector field $F=\left(f^{1}, \ldots, f^{n}\right) \in L^{p}\left(\mathbb{R}^{n}, \mathbb{R}^{n}\right)$. We first solve the Poisson equation

$$
F=\Delta U=\left(\Delta u^{1}, \ldots, \Delta u^{n}\right)
$$

for $U=\left(u^{1}, \ldots, u^{n}\right) \in \mathcal{D}^{\prime}\left(\mathbb{R}^{n}, \mathbb{R}^{n}\right)$. Note the identities

$$
\frac{\partial^{2} U}{\partial x_{i} \partial x_{j}}=-\mathbf{R}_{i j}(F) \in L^{p}\left(\mathbb{R}^{n} \times \mathbb{R}^{n}\right) \quad \text { for } i, j=1, \ldots, n,
$$

where $\mathbf{R}_{i j}=\mathbf{R}_{i} \circ \mathbf{R}_{j}$ are the second order Riesz transforms. Eq. (8.1) yields div-curl (also known as Hodge) decomposition of $F$.

$$
F=B+E,
$$

where

$$
B=\Delta U-\nabla \operatorname{div} U \quad \text { and } \quad E=\nabla \operatorname{div} U .
$$

These fields are easily seen to be divergence and curl free, respectively. More explicitely, with the aid of (8.2) we find that

$$
B=(\mathbf{I}+\mathbf{R} \otimes \mathbf{R}) F \quad \text { and } \quad E=-(\mathbf{R} \otimes \mathbf{R}) F .
$$

Hereafter, we use the notation $\mathbf{R} \otimes \mathbf{R}=\left[\mathbf{R}_{i j}\right]$ for the matrix of the second order Riesz transforms. We refer to the operators

$$
\mathbf{B}=\mathbf{I}+\mathbf{R} \otimes \mathbf{R} \quad \text { and } \quad \mathbf{E}=-\mathbf{R} \otimes \mathbf{R}
$$


as projections of $L^{p}\left(\mathbb{R}^{n}, \mathbb{R}^{n}\right)$ onto the spaces $\mathcal{B}^{p}\left(\mathbb{R}^{n}\right)$ and $\mathcal{E}^{p}\left(\mathbb{R}^{n}\right)$, respectively. For the use in the sequel we note that

$$
\operatorname{ker} \mathbf{B}=\mathcal{E}^{p}\left(\mathbb{R}^{n}\right) \quad \text { and } \quad \operatorname{ker} \mathbf{E}=\mathcal{B}^{p}\left(\mathbb{R}^{n}\right),
$$

which is clear from the uniqueness of the Hodge decomposition. Next, $F$ can also be written as

$$
F=\nabla(\operatorname{div} U)+\operatorname{div}(\operatorname{curl} U) .
$$

Here, we have applied the divergence operator to the matrix function curl $U$. The result is a vector field whose components are obtained by computing the divergence of the row vectors. Now, if $F \in \mathcal{D}^{\prime}\left(\mathbb{R}^{n}, \mathbb{R}^{n}\right)$ and curl $F$ happens to be in $L^{s}\left(\mathbb{R}^{n}, \mathbb{R}^{n \times n}\right)$, then we can solve Poisson's equation for $\operatorname{curl} U$, that is

$$
\Delta(\operatorname{curl} U)=\operatorname{curl}(\Delta U)=\operatorname{curl} F .
$$

Hence

$$
\frac{\partial^{2}(\operatorname{curl} U)}{\partial x_{i} \partial x_{j}}=-\mathbf{R}_{i j}(\operatorname{curl} F) \in L^{s}\left(\mathbb{R}^{n}, \mathbb{R}^{n \times n}\right) .
$$

In other words, the second term at (8.8) belongs to the Sobolev class $\mathcal{W}^{1, s}\left(\mathbb{R}^{n}, \mathbb{R}^{n}\right)$ while the first term, denoted by $F_{0}=\nabla(\operatorname{div} U)$, is a curl free distribution. We then conclude with the following Poincaré type Lemma:

LEMMA 8.1. - For each distribution $F \in \mathcal{D}^{\prime}\left(\mathbb{R}^{n}, \mathbb{R}^{n}\right)$, with curl $F \in L^{s}\left(\mathbb{R}^{n}, \mathbb{R}^{n \times n}\right)$ and $1<s<\infty$, there exists $E_{0} \in \mathcal{E}\left(\mathbb{R}^{n}, \mathbb{R}^{n}\right)$ such that $F-E_{0} \in \mathcal{W}^{1, s}\left(\mathbb{R}^{n}, \mathbb{R}^{n}\right)$. We also have a uniform bound

$$
\left\|F-E_{0}\right\|_{1, s}=\left\|D F-D E_{0}\right\|_{s} \leqslant C_{s}(n)\|\operatorname{curl} F\|_{s} .
$$

The following formula for the transposed differential of $F-E_{0}$ couldn't have been more explicit

$$
D^{t}\left(F-E_{0}\right)=(\mathbf{R} \otimes \mathbf{R})(\operatorname{curl} F) \in L^{s}\left(\mathbb{R}^{n}, \mathbb{R}^{n \times n}\right) .
$$

Here the notation $\mathbf{R} \otimes \mathbf{R}=\left[\mathbf{R}_{i j}\right]$ is being used for the operator acting on matrixfunctions, in analogy with the multiplication of matrices. We argue similarly for the dual statement:

LEMmA 8.2. - For each distribution $F \in \mathcal{D}^{\prime}\left(\mathbb{R}^{n}, \mathbb{R}^{n}\right)$, with $\operatorname{div} F \in L^{s}\left(\mathbb{R}^{n}, \mathbb{R}^{n}\right)$ and $1<s<\infty$, there exists $B_{0} \in \mathcal{B}\left(\mathbb{R}^{n}, \mathbb{R}^{n}\right)$ such that $F-B_{0} \in \mathcal{W}^{1, s}\left(\mathbb{R}^{n}, \mathbb{R}^{n}\right)$ and we have

$$
\left\|F-B_{0}\right\|_{1, s}=\left\|D F-D B_{0}\right\|_{s} \leqslant C_{s}(n)\|\operatorname{div} F\|_{s} .
$$

This follows from another explicit formula for the differential of $F-B_{0}$, namely

$$
D\left(F-B_{0}\right)=-(\mathbf{R} \otimes \mathbf{R})(\operatorname{div} F) .
$$


In each lemma, if the distribution $F$ happens to be in some space $L^{s^{\prime}}\left(\mathbb{R}^{n}, \mathbb{R}^{n}\right), 1<s^{\prime}<$ $\infty$, then so do the distributions $E_{0}$ and $B_{0}$. This is because both $E_{0}$ and $B_{0}$ can be expressed in terms of $F$ via Riesz transforms. Orlicz-Sobolev variants of these lemmas are also available, but we shall pursue this matter later. Concerning local fields, with the aid of formulas (2.1), we obtain

COROLlaRY 8.3. - Let $\mathcal{F}=[B, E]$ be a div-curl couple in $L_{\text {loc }}^{s}\left(\Omega, \mathbb{R}^{n} \times \mathbb{R}^{n}\right)$, $1<s<\infty$, and $\varphi \in C_{0}^{\infty}(\Omega)$. Then there exists a div-curl couple $\mathcal{F}_{0}=\left[B_{0}, E_{0}\right] \in$ $L^{s}\left(\mathbb{R}^{n}, \mathbb{R}^{n} \times \mathbb{R}^{n}\right)$ such that $\varphi \mathcal{F}-\mathcal{F}_{0} \in \mathcal{W}^{1, s}\left(\mathbb{R}^{n}, \mathbb{R}^{n}\right)$ and

$$
\left\|\varphi \mathcal{F}-\mathcal{F}_{0}\right\|_{1, s} \leqslant C(n, s)\||\nabla \varphi||\mathcal{F}|\|_{s} .
$$

As $\mathcal{F}_{0}$ is obtained via Riesz transforms of $\varphi \mathcal{F}$ it may be concluded that if $\mathcal{F} \in$ $L_{\text {loc }}^{s^{\prime}}\left(\Omega, \mathbb{R}^{n} \times \mathbb{R}^{n}\right)$ for some other exponent $1<s^{\prime}<\infty$, then $\mathcal{F}_{0} \in L^{s^{\prime}}\left(\mathbb{R}^{n}, \mathbb{R}^{n} \times \mathbb{R}^{n}\right)$. Furthermore, we have the following singular integral expressions for the differential of $\varphi \mathcal{F}-\mathcal{F}_{0}$ :

$$
\begin{aligned}
& D\left(\varphi B-B_{0}\right)=-(\mathbf{R} \otimes \mathbf{R})\langle\nabla \varphi, B\rangle, \\
& D^{t}\left(\varphi E-E_{0}\right)=(\mathbf{R} \otimes \mathbf{R})[E * \nabla \varphi] .
\end{aligned}
$$

In either of these expressions we notice that

$$
\int_{\mathbb{R}^{n}}\langle\nabla \varphi, B\rangle=0 \quad \text { and } \quad \int_{\mathbb{R}^{n}}[E * \nabla \varphi]=0 .
$$

Hence, if $\Omega$ is a cube or a ball centered at $x_{0} \in \mathbb{R}^{n}$, then for $x \in \mathbb{R}^{n}-2 \Omega$ we have the point-wise inequality

$$
\left|D\left(\varphi \mathcal{F}-\mathcal{F}_{0}\right)(x)\right| \leqslant \frac{C(n) \operatorname{diam} \Omega}{\left|x-x_{0}\right|^{n+1}}\||\nabla \varphi||\mathcal{F}|\|_{L^{1}\left(\mathbb{R}^{n}\right)} .
$$

This also gives

$$
\left|\left(\varphi \mathcal{F}-\mathcal{F}_{0}\right)(x)\right| \leqslant \frac{C(n) \operatorname{diam} \Omega}{\left|x-x_{0}\right|^{n}}\||\nabla \varphi||\mathcal{F}|\|_{L^{1}\left(\mathbb{R}^{n}\right)}
$$

\section{Hilbert transform}

When studying quasiconformal mappings in the complex plane an analogue of the Hilbert transform was introduced by Beurling, nowadays known as the Beurling-Ahlfors transform. This is a singular integral operator of the form

$$
(\mathbf{A} f)(z)=\frac{1}{2 \pi i} \iint_{\mathbb{C}} \frac{f(\xi) d \xi \wedge d \bar{\xi}}{(z-\xi)^{2}}, \quad f \in L^{p}(\mathbb{C}), 1<p<\infty .
$$

We wish to argue by analogy with this operator and its higher dimensional counterparts introduced in [41]. 
Let $F \in L^{p}\left(\mathbb{R}^{n}, \mathbb{R}^{n}\right), 1<p<\infty$, be a given vector field. We decompose it as $F=B+E$, where $B \in \mathcal{B}^{p}\left(\mathbb{R}^{n}\right)$ and $E \in \mathcal{E}^{p}\left(\mathbb{R}^{n}\right)$. Then we set $\mathbf{S}(F)=E-B$. In terms of the projection operators, we can write

$$
-\mathbf{S}=\mathbf{B}-\mathbf{E}=\mathbf{I}+2 \mathbf{R} \otimes \mathbf{R} .
$$

This operator in dimension 2 is not exactly the same as A. Nevertheless, it captures basic algebraic and analytic features of $\mathbf{A}$. Before discussing more advanced results, let us list some properties of particular interest

(i) $\mathbf{S}$ is an involution, that is, $\mathbf{S} \circ \mathbf{S}=\mathbf{I}$;

(ii) $\mathbf{S}$ is self adjoint, that is

$$
\int_{\mathbb{R}^{n}}\langle\mathbf{S} F, G\rangle=\int_{\mathbf{R}^{n}}\langle F, \mathbf{S} G\rangle
$$

for $F \in L^{p}\left(\mathbb{R}^{n}, \mathbb{R}^{n}\right)$ and $G \in L^{q}\left(\mathbb{R}^{n}, \mathbb{R}^{n}\right)$, with $1<p, q<\infty, p+q=p q$.

Thus, for $p=q=2$, we find that

(iii) $\mathbf{S}: L^{2}\left(\mathbb{R}^{n}, \mathbb{R}^{n}\right) \rightarrow L^{2}\left(\mathbb{R}^{n}, \mathbb{R}^{n}\right)$ is an isometry.

This operator seems to be an excellent generalization of the Hilbert transform on the real line. When raised to the level of differential forms one obtains even more general operator $\mathbf{S}: L^{p}\left(\mathbb{R}^{n}, \Lambda^{l}\right) \rightarrow L^{p}\left(\mathbb{R}^{n}, \Lambda^{l}\right)$. The latter has proven to be extremely useful in the study of quasiconformal mappings [26,41]. But the calculation of its $p$-norms remains one of the outstanding problems in the area, even in the case $n=2$. There have been several attempts to identify these norms $[42,6,5,38]$, which have led to the following

CONJECTURE 9.1. - For all dimensions $n \geqslant 2$ and $1<p<\infty$, we have

$$
\|\mathbf{S}\|_{p}=\max \left\{p-1, \frac{1}{p-1}\right\} .
$$

See next section for much more general setting.

So far, the best estimate belongs to R. Bañuelos and A. Lindeman [6], which asserts that

$$
\|\mathbf{S}\|_{p} \leqslant 6 \max \left\{p-1, \frac{1}{p-1}\right\} .
$$

Note that this estimate is dimension free, but, in view of the property (iii) it does not exhibit correct upper bound for $p$ close to 2. Riesz-Thorin Interpolation Theorem comes to the rescue, details being left for the reader.

LEMMA 9.1. - For a pair $1<q \leqslant 2 \leqslant p$ of Hölder conjugate exponents, $p+q=p q$, we have

$$
\|\mathbf{S}\|_{q}=\|\mathbf{S}\|_{p} \leqslant 7 p-13 .
$$

Let us point out that the equation $\|\mathbf{S}\|_{q}=\|\mathbf{S}\|_{p}$ is straightforward by a duality argument.

We have reserved our final section of Part One for comments concerning possible extensions of these ideas. 


\section{The elliptic complex setting}

There is always further to go. One way is by looking at the exact sequences of first order differential operators, generalizing the previously considered sequence

$$
\mathcal{D}^{\prime}\left(\mathbb{R}^{n}, \mathbb{R}\right) \stackrel{\nabla}{\longrightarrow} \mathcal{D}^{\prime}\left(\mathbb{R}^{n}, \mathbb{R}^{n}\right) \stackrel{\text { curl }}{\longrightarrow} \mathcal{D}^{\prime}\left(\mathbb{R}^{n}, \mathbb{R}^{n \times n}\right) .
$$

Let $\Lambda_{-}, \Lambda$ and $\Lambda_{+}$be arbitrary finite dimensional inner product spaces. Consider a short elliptic complex

$$
\mathcal{D}^{\prime}\left(\mathbb{R}^{n}, \Lambda_{-}\right) \stackrel{D_{-}}{\longrightarrow} \mathcal{D}^{\prime}\left(\mathbb{R}^{n}, \Lambda\right) \stackrel{D_{+}}{\longrightarrow} \mathcal{D}^{\prime}\left(\mathbb{R}^{n}, \Lambda_{+}\right),
$$

where $D_{-}$and $D_{+}$are first order partial differential operators with constant coefficients. More precisely, the sequence of symbols

$$
\Lambda_{-} \stackrel{D_{-}(\xi)}{\longrightarrow} \Lambda \stackrel{D_{+}(\xi)}{\longrightarrow} \Lambda_{+} \quad \text { for } \xi \in \mathbb{R}^{n}-\{0\}
$$

is supposed to be exact; $\operatorname{Im} D_{-}(\xi)=\operatorname{ker} D_{+}(\xi)$.

Note that the dual complex

$$
\mathcal{D}^{\prime}\left(\mathbb{R}^{n}, \Lambda_{-}\right) \stackrel{D_{-}^{*}}{\longleftarrow} \mathcal{D}^{\prime}\left(\mathbb{R}^{n}, \Lambda\right) \stackrel{D_{+}^{*}}{\longleftarrow} \mathcal{D}^{\prime}\left(\mathbb{R}^{n}, \Lambda_{+}\right)
$$

is elliptic if and only if the original complex is. Then the elliptic second order operator

$$
\Delta=D_{-} D_{-}^{*}+D_{+}^{*} D_{+}: \mathcal{D}^{\prime}\left(\mathbb{R}^{n}, \Lambda\right) \rightarrow \mathcal{D}^{\prime}\left(\mathbb{R}^{n}, \Lambda\right)
$$

is a natural tool to various estimates.

With the aid of singular integrals one can solve the Poisson equation

$$
\Delta U=F \in L^{p}\left(\mathbb{R}^{n}, \Lambda\right), \quad 1<p<\infty,
$$

uniquely for $U \in \mathcal{W}^{2, p}\left(\mathbb{R}^{n}, \Lambda\right)$. The basic estimate is

$$
\left\|\frac{\partial^{2} U}{\partial x_{i} \partial x_{j}}\right\|_{p} \leqslant C_{p}\|F\|_{p}, \quad i, j=1, \ldots, n .
$$

This gives rise to the Hodge decomposition of a vector field $F \in L^{p}\left(\mathbb{R}^{n}, \Lambda\right)$, namely

$$
F=D_{-} \alpha+D_{+}^{*} \beta
$$

with $\alpha \in \mathcal{W}^{1, p}\left(\mathbb{R}^{n}, \Lambda_{-}\right)$and $\beta \in \mathcal{W}^{1, p}\left(\mathbb{R}^{n}, \Lambda_{+}\right)$, which are explicitely expressed in terms of the first order derivatives of $U$. Then the Hilbert transform is defined by the rule

$$
\mathbf{S} F=D_{-} \alpha-D_{+}^{*} \beta, \quad \mathbf{S}: L^{p}\left(\mathbb{R}^{n}, \Lambda\right) \rightarrow L^{p}\left(\mathbb{R}^{n}, \Lambda\right), \quad 1<p<\infty .
$$


On the analogy of the div-curl couples one might consider the pairs $\mathcal{F}=[X, Y] \in$ $L^{p}\left(\mathbb{R}^{n}, \Lambda \times \Lambda\right)$, with $X=D_{-} \alpha$ and $Y=D_{+}^{*} \beta$, and also their Jacobian $J(x, \mathcal{F})=$ $\langle X(x), Y(x)\rangle$.

We trust that the further resemblance to the quasiharmonic fields is selfexplanatory.

Finding the norms of the Hilbert transform associated with the elliptic complex (10.2) is central to the $L^{p}$-theory of PDEs. Numerous examples suggest the following

CONJECTURE 10.1. - For a Hölder conjugate pair $1<q \leqslant p<\infty, p+q=p q$, we have

$$
\|\mathbf{S}\|_{q}=\|\mathbf{S}\|_{p}=p-1 .
$$

The expected lower bound for $\|\mathbf{S}\|_{p}$ is easily seen to be true. What remains is to show the estimate

$$
\left\|\mathcal{F}^{+}\right\|_{p} \leqslant(p-1)\left\|\mathcal{F}^{-}\right\|_{p}, \quad 2 \leqslant p<\infty .
$$

Without getting into technicalities, this reduces to showing that

$$
I[\mathcal{F}]=\int_{\mathbb{R}^{n}}\left((p-1)\left|\mathcal{F}^{-}\right|-\left|\mathcal{F}^{+}\right|\right)\left(\left|\mathcal{F}^{-}\right|+\left|\mathcal{F}^{+}\right|\right)^{p-1} \geqslant 0,
$$

see D. Burkholder [11] and [38,5,2] for further developments. It is in this way that we become interested in the variational integrals of the form

$$
I[\mathcal{F}]=\int_{\mathbb{R}_{n}} \Phi(\mathcal{F}(x)) d x,
$$

where $\Phi$ is a continuous function on $\Lambda$. Such integrals are well defined on the pairs

$$
\mathcal{F}=[X, Y] \in C_{0}^{\infty}\left(\mathbb{R}^{n}, \Lambda \times \Lambda\right) .
$$

We say that $\Phi$ is quasiconvex if

$$
\int_{\mathbb{R}^{n}}\left[\Phi\left(\mathcal{F}_{0}+\mathcal{F}(x)\right)-\Phi\left(\mathcal{F}_{0}\right)\right] d x \geqslant 0
$$

for every $\mathcal{F}_{0} \in \Lambda \times \Lambda$ and $\mathcal{F}(x)=[X(x), Y(x)]$, whenever $X \in D_{-} C_{0}^{\infty}\left(\mathbb{R}^{n}, \Lambda_{-}\right)$and $Y \in D_{+}^{*} C_{0}^{\infty}\left(\mathbb{R}^{n}, \Lambda_{+}\right)$.

It is of great interest to characterize quasiconvexity by imposing only algebraic conditions on the integrand $\Phi$. Note that Burkholder's functional (10.12) is convex in the so-called singular directions, zeros of the Jacobian. For a general functional (10.13), this simply means that the real variable function $t \mapsto \Phi\left(\mathcal{F}_{0}+t \mathcal{F}\right)$ with $\mathcal{F}_{0} \in \Lambda \times \Lambda$ and $\mathcal{F}=$ $[X, Y]$ is convex, whenever $X$ and $Y$ are orthogonal vectors in $\Lambda$. If we confine ourselves to the elliptic complexes such that $\bigcup_{|\xi|=1} \operatorname{ker} D_{+}(\xi)=\Lambda$, then condition (10.14) implies convexity in singular directions, see the forthcoming paper [33]. A puzzling thing about an analogous conjecture in the multidimensional Calculus of Variations is its failure, for 
$n \geqslant 3$, as shown by V. Šverák [71]. Conjecture 10.2 is likely to be true in all dimensions since it assumes convexity in more directions.

For relevant literature we refer to [20]. We are now left with a challenging conjecture for the converse:

CONJECTURE 10.2. - Convexity in singular directions implies quasiconvexity.

\section{Part Two:}

\section{Bounded Distortion}

This part is dedicated to quasiharmonic fields of bounded distortion, say

$$
1 \leqslant K(x) \leqslant K \text { a.e. }
$$

Just as quasiconformal mappings in the plane, the $K$-quasiharmonic fields will be used for the study of the second order elliptic equations in $\mathbf{R}^{n}$.

\section{The Beltrami operator and critical exponents}

Let $\mu$ be a measurable function in $\mathbb{R}^{n}$ valued in symmetric matrices such that

$$
\|\mu(x)\|=\max _{|\xi|=1}|\mu(x) \xi| \leqslant \frac{K(x)-1}{K(x)+1},
$$

where $1 \leqslant K(x)<\infty$ a.e. The central question that we want to address here is the invertibility of the Beltrami operator

$$
\mathbf{I}-\mu \mathbf{S}: L^{p}\left(\mathbb{R}^{n}, \mathbb{R}^{n}\right) \rightarrow L^{p}\left(\mathbb{R}^{n}, \mathbb{R}^{n}\right), \quad 1<p<\infty,
$$

and its adjoint

$$
\mathbf{I}-\mathbf{S} \mu: L^{q}\left(\mathbb{R}^{n}, \mathbb{R}^{n}\right) \rightarrow L^{q}\left(\mathbb{R}^{n}, \mathbb{R}^{n}\right), \quad p+q=p q .
$$

The latter being characterized by the identity

$$
\int_{\mathbb{R}^{n}}\langle(\mathbf{I}-\mu \mathbf{S}) F, G\rangle=\int_{\mathbb{R}^{n}}\langle F,(\mathbf{I}-\mathbf{S} \mu) G\rangle
$$

for $F \in L^{p}\left(\mathbb{R}^{n}, \mathbb{R}^{n}\right)$ and $G \in L^{q}\left(\mathbb{R}^{n}, \mathbb{R}^{n}\right)$. Basic estimates of $\mathbf{I}-\mathbf{S} \mu$ can be obtained from those of $\mathbf{I}-\mu \mathbf{S}$ via the identity

$$
\mathbf{I}-\mathbf{S} \mu=\mathbf{S} \circ(\mathbf{I}-\mu \mathbf{S}) \circ \mathbf{S} .
$$

Both operators are certainly invertible in $L^{p}\left(\mathbb{R}^{n}, \mathbb{R}^{n}\right)$ and $L^{q}\left(\mathbb{R}^{n}, \mathbb{R}^{n}\right)$, for $1<q \leqslant p$ $<\infty$, with $p+q=p q$, such that

$$
\|\mu\|_{\infty}\|\mathbf{S}\|_{q}=\|\mu\|_{\infty}\|\mathbf{S}\|_{p}<1 .
$$


Recall that our basic assumption is $1 \leqslant K(x) \leqslant K$. To each such constant $K$ we associate a pair of Hölder conjugate exponents, $1 \leqslant q_{K}<2<p_{K} \leqslant \infty$, determined implicitely by the equation

$$
\|\mathbf{S}\|_{q_{K}}=\|\mathbf{S}\|_{p_{K}}=\frac{K+1}{K-1} .
$$

As $\|\mathbf{S}\|_{2}=1$, standard interpolation arguments give strict inequality

$$
\|\mathbf{S}\|_{r}<\frac{K+1}{K-1} \quad \text { for all } r \in\left(q_{K}, p_{K}\right) .
$$

Thus the critical interval $\left(q_{K}, p_{K}\right)$ consists of the exponents $r$ for which the operators $\mathbf{I}-\mu \mathbf{S}$ and $\mathbf{I}-\mathbf{S} \mu$ are invertible in $L^{r}\left(\mathbb{R}^{n}, \mathbb{R}^{n}\right)$. To facilitate explicit estimates we record the following dimension free bounds for the critical exponents

$$
q_{K}<\frac{14 K-12}{7 K-5}<2<\frac{14 K-12}{7 K-7}<p_{K}, \quad \text { for } K>1
$$

which is immediate from Lemma 9.2. An affirmative answer to Conjecture 9.1 would yield invertibility of the operators

$$
\mathbf{I}-\mu \mathbf{S}, \quad \mathbf{I}-\mathbf{S} \mu: L^{r}\left(\mathbb{R}^{n}, \mathbb{R}^{n}\right) \rightarrow L^{r}\left(\mathbb{R}^{n}, \mathbb{R}^{n}\right)
$$

for all exponents $r$ in the interval

$$
\frac{2 K}{K+1}<r<\frac{2 K}{K-1}
$$

As an interesting note this is true in dimension 2 [4], though the $p$-norms of $\mathbf{S}$ still remain unknown.

\section{Caccioppoli inequalities}

Our next objective is to estimate div-curl solutions of the nonhomogeneous Beltrami equation

$$
\mathcal{F}^{-}(x)=\mu(x) \mathcal{F}^{+}(x)+G(x) .
$$

We will be working under the assumption that $\mathcal{F} \in L_{\text {loc }}^{s^{\prime}}\left(\Omega, \mathbb{R}^{n} \times \mathbb{R}^{n}\right)$ for at least one exponent $s^{\prime}$ in the critical interval $\left(q_{K}, p_{K}\right)$. Fix an exponent $r$ such that

$$
\max \left\{q_{K}, \frac{n}{n-1}\right\}<r<p_{K} .
$$

The following Caccioppoli type estimate holds

PROPOSITION 12.1. - Suppose that $G \in L_{\text {loc }}^{r}\left(\Omega, \mathbb{R}^{n}\right)$ and $\mathcal{F} \in L_{\text {loc }}^{\frac{n r}{n+r}}\left(\Omega, \mathbb{R}^{n} \times \mathbb{R}^{n}\right)$ satisfy (12.1). Then $\mathcal{F} \in L_{\text {loc }}^{r}\left(\Omega, \mathbb{R}^{n} \times \mathbb{R}^{n}\right)$ and for each test function $\varphi \in C_{0}^{\infty}(\Omega)$ 
we have

$$
\|\varphi \mathcal{F}\|_{r} \leqslant C(n, K)\|\varphi G\|_{r}+C(n, K)\||\nabla \varphi||\mathcal{F}|\|_{\frac{n r}{n+r}} .
$$

Proof. - Multiplying (12.1) by $\varphi$ leads to an equation in the entire space $\mathbb{R}^{n}$

$$
\left(\varphi \mathcal{F}^{-}\right)=\mu(\varphi \mathcal{F})^{+}+\varphi G,
$$

where $\mu: \mathbb{R}^{n} \rightarrow \mathbb{R}^{n \times n}$ is defined to be zero outside $\Omega$. Although $\varphi \mathcal{F}$ is no longer a divcurl couple we can find one which is close to $\varphi \mathcal{F}$. Indeed, by Corollary 8.3 there exists a div-curl couple

$$
\mathcal{F}_{0} \in L^{\frac{n r}{n+r}}\left(\mathbb{R}^{n}, \mathbb{R}^{n} \times \mathbb{R}^{n}\right) \cap L^{s^{\prime}}\left(\mathbb{R}^{n}, \mathbb{R}^{n} \times \mathbb{R}^{n}\right),
$$

which satisfies

$$
\left\|\varphi \mathcal{F}-\mathcal{F}_{0}\right\|_{1, \frac{n r}{n+r}} \leqslant C(n, r)\||\nabla \varphi||\mathcal{F}|\|_{\frac{n r}{n+r}} .
$$

For the couple $\Phi=\varphi \mathcal{F}-\mathcal{F}_{0}$, one may use Sobolev's theorem to find that $\Phi \in$ $L^{r}\left(\mathbb{R}^{n}, \mathbb{R}^{n} \times \mathbb{R}^{n}\right)$ and its norm is controlled by

$$
\|\Phi\|_{r} \leqslant C(n, r)\||\nabla \varphi||\mathcal{F}|\|_{\frac{n r}{n+r}} .
$$

Eq. (12.4) takes the form

$$
\mathcal{F}_{0}^{-}-\mu \mathcal{F}_{0}^{+}=\varphi G+\mu \Phi^{+}-\Phi^{-}
$$

or, equivalently

$$
(\mathbf{I}-\mu \mathbf{S}) \mathcal{F}_{0}^{-}=\varphi G+\mu \Phi^{+}-\Phi^{-} .
$$

The right hand side belongs to $L^{r}\left(\mathbb{R}^{n}, \mathbb{R}^{n}\right)$, while $\mathcal{F}_{0}^{-} \in L^{s^{\prime}}\left(\mathbb{R}^{n}, \mathbb{R}^{n}\right)$. Both exponents $r$ and $s^{\prime}$ are in the critical interval $\left(q_{K}, p_{K}\right)$. It is at this point that we make use of the inverse operator $(\mathbf{I}-\mu \mathbf{S})^{-1}: L^{s}\left(\mathbb{R}^{n}, \mathbb{R}^{n}\right) \rightarrow L^{s}\left(\mathbb{R}^{n}, \mathbb{R}^{n}\right)$. We recall that this operator exists and is bounded whenever $q_{K}<s<p_{K}$. Consequently, $\mathcal{F}_{0}^{-}$belongs to $L^{r}\left(\mathbb{R}^{n}, \mathbb{R}^{n}\right)$ and so does $\mathcal{F}_{0}^{+}=\mathbf{S}\left(\mathcal{F}_{0}^{-}\right)$. Moreover, we have

$$
\left\|\mathcal{F}_{0}\right\|_{r} \leqslant C(n, K)\left\|\varphi G+\mu \Phi^{+}-\Phi^{-}\right\|_{r} .
$$

Hence $\varphi \mathcal{F}=\Phi+\mathcal{F}_{0} \in L^{r}\left(\mathbb{R}^{n}, \mathbb{R}^{n} \times \mathbb{R}^{n}\right)$, and by this latter estimate it follows that

$$
\|\varphi \mathcal{F}\|_{r}=\left\|\Phi+\mathcal{F}_{0}\right\|_{r} \leqslant C(n, K)\|\Phi\|_{r}+C(n, K)\|\varphi G\|_{r} .
$$

When combined with (12.6) this implies the inequality (12.3), completing the proof of Proposition 12.1.

Now the proof of Theorem 2 is straightforward. Indeed, for $K$-quasiharmonic fields we have Eq. (12.1) with $G \equiv 0$ in (12.1). Proposition 12.1 gives Caccioppoli type inequality

$$
\|\varphi \mathcal{F}\|_{r} \leqslant C(n, K)\||\nabla \varphi| \mathcal{F}\|_{\frac{n r}{n+r}} .
$$


Repeated application of this estimate results in the improvement of the degree of integrability of $\mathcal{F}$, exactly as stated in part (a) of Theorem 2 . The second part is immediate, details being left to the reader.

One inference from (12.8) is the reverse Hölder inequality for integral means over the cubes $Q$ in the domain $\Omega$

$$
\left(f_{Q}|\mathcal{F}|^{r}\right)^{\frac{1}{r}} \leqslant C(n, K)\left(f_{2 Q}|\mathcal{F}|^{\frac{n r}{n+r}}\right)^{\frac{n+r}{n r}} .
$$

Here, the double cube, denoted by $2 Q$, is also assumed to be contained in $\Omega$.

\section{An estimate for nonhomogeneous equations}

We now turn to an elliptic PDE of the form

$$
\operatorname{div} A(x) \nabla u=\operatorname{div} F \quad \text { in } \Omega \subset \mathbb{R}^{n},
$$

where $F$ is a given vector field in $L_{l o c}^{r}\left(\Omega, \mathbb{R}^{n}\right)$ and the coefficient matrix verifies

$$
\frac{1}{K}|\xi|^{2} \leqslant\langle A(x) \xi, \xi\rangle \leqslant K|\xi|^{2}
$$

The div-curl couple $\mathcal{F}=[B, E]=[A(x) \nabla u-F, \nabla u]$ solves a nonhomogeneous Beltrami equation

$$
\mathcal{F}^{-}(x)=\mu(x) \mathcal{F}^{+}(x)+G(x) .
$$

Here the Beltrami matrix is explicitely expressed in terms of $A$

$$
\mu(x)=\frac{\mathbf{I}-A(x)}{\mathbf{I}+A(x)}
$$

while the vector field $G$ is given in terms of $F$ by

$$
G(x)=[\mathbf{I}+A(x)]^{-1} F .
$$

As the eigenvalues of $A(x)$ lie between $K^{-1}$ and $K$ it follows that

$$
\|\mu(x)\| \leqslant \sup _{K^{-1} \leqslant \lambda \leqslant K}\left|\frac{1-\lambda}{1+\lambda}\right|=\frac{K-1}{K+1} .
$$

This makes it legitimate to apply Proposition 12.1 . We forgo possibly more general implications, just only extract the following useful estimate:

Proposition 13.1. - Suppose $u \in W_{\text {loc }}^{1,2}(\Omega)$ satisfies Eq. (13.1) with $F \in L_{l o c}^{r}(\Omega$, $\left.\mathbb{R}^{n}\right)$, where $2 \leqslant r<p_{K}$. Then $u \in W_{\text {loc }}^{1, r}(\Omega)$ and

$$
\|\varphi \nabla u\|_{r} \leqslant C(n, K)\|\varphi F\|_{r}+C(n, K)\||\nabla \varphi||\nabla u|\|_{\frac{n r}{n+r}} .
$$


Recall that $P_{K}$ is not smaller than $2+\frac{2}{7(K-1)}$.

\section{The Dirichlet problem in the range of critical exponents}

This section is devoted to a qualitative analysis of the boundary value problem

$$
\left\{\begin{array}{l}
\sum_{i j=1} \frac{\partial}{\partial x_{i}}\left(A_{i j}(x) \frac{\partial u}{\partial x_{j}}\right)=\operatorname{div} F \\
u \in W_{0}^{1, r}(\Omega)
\end{array}\right.
$$

where $F \in L^{r}\left(\Omega, \mathbb{R}^{n}\right)$ and $A$ verifies the usual ellipticity bounds

$$
K^{-1}|\xi|^{2} \leqslant\langle A(x) \xi, \xi\rangle \leqslant K|\xi|^{2} .
$$

The natural space here is of course $W_{0}^{1,2}(\Omega)$. The case $r=2$ poses no difficulty since the existence and uniqueness follow by variational principles. We have

$$
\|\nabla u\|_{2} \leqslant K\|F\|_{2} \text {. }
$$

Thus Eq. (14.1) defines a bounded operator $\mathbf{A}: L^{2}\left(\Omega, \mathbb{R}^{n}\right) \rightarrow L^{2}\left(\Omega, \mathbb{R}^{n}\right)$ which takes a given vector field $F$ into the gradient of the solution. Like for the Laplace operator, the $L^{r}$-bounds with $r \neq 2$ need some regularity of $\Omega$. For the sake of simplicity we confine ourselves to only three types of domains: $\Omega=\mathbb{R}^{n}, \Omega=\mathbb{R}_{+}^{n}$ and $\Omega$ is a cube in $\mathbb{R}^{n}$.

THEOREM 14.1. - The operator A extends continuously to $L^{r}\left(\Omega, \mathbb{R}^{n}\right)$ for all exponents $r$ in the interval (1.21).

Proof. - It suffices to prove the uniform estimate

$$
\|\nabla u\|_{r} \leqslant C(n, K)\|F\|_{r}
$$

We shall derive it from the Beltrami equation (13.2). For $\Omega=\mathbb{R}^{n}$ there is no need to multiply this equation by a test function $\varphi \in C_{0}^{\infty}\left(\mathbb{R}^{n}\right)$. In this case estimate (12.3) remains valid with $\varphi \equiv 1$. Hence (14.4) is immediate.

We next consider the half space $\Omega=\mathbb{R}_{+}^{n}=\left\{\left(x_{1}, \ldots, x_{n}\right) ; x_{n}>0\right\}$. Let $\rho: \mathbb{R}^{n} \rightarrow$ $\mathbb{R}^{n}$ denote the reflection about the hyperplane $x_{n}=0$, that is, $\rho\left(x_{1}, \ldots, x_{n-1}, x_{n}\right)=$ $\left(x_{1}, \ldots, x_{n-1},-x_{n}\right)$. The task is now to extend equation (14.1) to $\mathbb{R}^{n}$. We define $u(x)=-u(\rho x)$ for $x \in \mathbb{R}_{-}^{n}$. Obviously $\nabla u(x)=-(\rho \nabla u)(\rho x)$ for $x \in \mathbb{R}_{-}^{n}$. Hence, $u \in W^{1, r}\left(\mathbb{R}^{n}\right)$ and we have

$$
\int_{\mathbb{R}^{n}}|\nabla u(x)|^{r} d x=2 \int_{\mathbb{R}_{+}^{n}}|\nabla u(x)|^{r} d x .
$$


Guided by the above extension of $\nabla u$ we find out how to extend $F$ to $\mathbb{R}^{n}$. Likewise, $F(x)=-\rho F(\rho x)$ for $x \in \mathbb{R}^{n}$. Hence

$$
\int_{\mathbb{R}^{n}}|F(x)|^{r} d x=2 \int_{\mathbb{R}_{+}^{n}}|F(x)|^{r} d x .
$$

The coefficient matrix $A=A(x)$ can be extended to $\mathbb{R}^{n}$ by setting $A(x)=A(\rho x)$ for $x \in \mathbb{R}_{-}^{n}$, which leads to the same ellipticity bounds as in (14.2), for all $x \in \mathbb{R}^{n}$. Although it is not obvious at this point it is nevertheless true that

$$
\operatorname{div} A(x) \nabla u=\operatorname{div} F \quad \text { in } \mathbb{R}^{n}
$$

in the sense of distributions. On account of the already established estimate in $\mathbb{R}^{n}$, we conclude with the inequality

$$
\begin{aligned}
\|\nabla u\|_{L^{r}\left(\mathbb{R}_{+}^{n}\right)} & =\left(\frac{1}{2}\right)^{1 / r}\|\nabla u\|_{L^{r}\left(\mathbb{R}^{n}\right)} \leqslant\left(\frac{1}{2}\right)^{1 / r} C(n, K)\|F\|_{L^{r}\left(\mathbb{R}^{n}\right)} \\
& =C(n, K)\|F\|_{L^{r}\left(\mathbb{R}_{+}^{n}\right)}
\end{aligned}
$$

with the same constant as for the entire space $\mathbb{R}^{n}$.

The case when $\Omega$ is a regular bounded domain, such as a cube or ball in $\mathbb{R}^{n}$, needs handling with additional arguments. We begin, as before, by extending Eq. (14.1) slightly beyond $\partial \Omega$. For instance if $\Omega$ is a cube one can reflect $u, F$ and the coefficient matrix $A$ across the faces of $\Omega$ in much the same way as we did for $\mathbb{R}_{+}^{n}$. New cubes emerge in this process and we continue reflecting across their faces. At the end we look at $u$ as a local solution to the extended equation in the double cube

$$
\operatorname{div} A(x) \nabla u=\operatorname{div} F \quad \text { in } 2 \Omega .
$$

The point is that the norms of $\nabla u$ and $F$ are controlled by those over the original domain, namely

$$
\|\nabla u\|_{L^{s}(2 \Omega)} \leqslant C(n, K)\|\nabla u\|_{L^{s}(\Omega)}
$$

and

$$
\|F\|_{L^{s}(2 \Omega)} \leqslant C(n, K)\|F\|_{L^{s}(\Omega)}
$$

for all $1 \leqslant s \leqslant r$.

Let us first assume that $2 \leqslant r<p_{K}$. Proposition 13.1 applies to the cutoff function $\varphi \in C_{0}^{\infty}(2 \Omega)$ such that $0 \leqslant \varphi(x) \leqslant 1, \varphi(x) \equiv 1$ on $\Omega$ and $|\nabla \varphi(x)| \leqslant C(n) \operatorname{diam}^{-1}(\Omega)$. It results in the following estimate

$$
\begin{aligned}
\left(f_{\Omega}|\nabla u|^{r}\right)^{\frac{1}{r}} & \leqslant C(n, K)\left(f_{2 \Omega}|\nabla u|^{\frac{n r}{n+r}}\right)^{\frac{n+r}{n r}}+C(n, K)\left(f_{2 \Omega}|F|^{r}\right)^{\frac{1}{r}} \\
& \leqslant C(n, K)\left(f_{\Omega}|\nabla u|^{\frac{n r}{n+r}}\right)^{\frac{n+r}{n r}}+C(n, K)\left(f_{\Omega}|F|^{r}\right)^{\frac{1}{r}} .
\end{aligned}
$$


The latter are due to (14.10) and (14.11). Applying Hölder's and then Young's inequality we see that for every positive $\varepsilon$

$$
\begin{aligned}
\left(f_{\Omega}|\nabla u|^{\frac{n r}{n+r}}\right)^{\frac{n+r}{n r}} & \leqslant\left(f_{\Omega}|\nabla u|^{r}\right)^{\frac{n-1}{n r}}\left(f_{\Omega}|\nabla u|^{2}\right)^{\frac{1}{2 n}} \\
& \leqslant \frac{n-1}{n} \varepsilon\left(f_{\Omega}|\nabla u|^{r}\right)^{\frac{1}{r}}+\frac{1}{n \varepsilon^{n-1}}\left(f_{\Omega}|\nabla u|^{2}\right)^{\frac{1}{2}} .
\end{aligned}
$$

On substituting this into (14.12) with $\varepsilon$ sufficiently small we obtain

$$
\left(f_{\Omega}|\nabla u|^{r}\right)^{1 / r} \leqslant C(n, K)\left(f_{\Omega}|F|^{r}\right)^{1 / r}+C(n, K)\left(f_{\Omega}|\nabla u|^{2}\right)^{1 / 2} .
$$

On the other hand, the $L^{2}$-bound at (14.3) yields

$$
\left(f_{\Omega}|\nabla u|^{2}\right)^{1 / 2} \leqslant K\left(f_{\Omega}|F|^{2}\right)^{1 / 2} \leqslant K\left(f_{\Omega}|F|^{r}\right)^{1 / r} .
$$

Hence the desired estimate (14.4) follows.

By duality, we can easily pass to the case $q_{K}<r \leqslant 2$. Here are some details. Let $r^{\prime}$ denote the Hölder conjugate to $r$, which is obviously in the interval $2 \leqslant r^{\prime}<p_{K}$. According to the previous case, we can solve uniquely the equation $\operatorname{div}\left(A \nabla u^{\prime}\right)=\operatorname{div} F^{\prime}$ for a function $u^{\prime} \in W_{0}^{1, r^{\prime}}(\Omega)$, where $F^{\prime}=|\nabla u|^{r-2} \nabla u$ is regarded as a given vector field in $L^{r^{\prime}}\left(\Omega, \mathbb{R}^{n}\right)$. On account of the already established estimate (14.4), with exponent $r^{\prime}$ in place of $r$, we can write

$$
\begin{aligned}
\int_{\Omega}|\nabla u|^{r} & =\int_{\Omega}\left\langle\nabla u, F^{\prime}\right\rangle=\int_{\Omega}\left\langle\nabla u, A \nabla u^{\prime}\right\rangle \\
& =\int_{\Omega}\left\langle A \nabla u, \nabla u^{\prime}\right\rangle=\int_{\Omega}\left\langle F, \nabla u^{\prime}\right\rangle \leqslant\|F\|_{r}\left\|\nabla u^{\prime}\right\|_{r^{\prime}} \\
& \leqslant C(n, K)\|F\|_{r}\left\|F^{\prime}\right\|_{r^{\prime}}=C(n, K)\|F\|_{r}\|\nabla u\|_{r}^{r-1} .
\end{aligned}
$$

Hence the desired inequality

$$
\|\nabla u\|_{r} \leqslant C(n, K)\|F\|_{r} .
$$

\section{Measure in the right hand side}

This section will be devoted to the equation

$$
\operatorname{div} A(x) \nabla u=v,
$$

where $v$ is a Radon (signed) measure of finite total variation in a cube $\Omega \subset \mathbb{R}^{n}$. Some novelties ought to be mentioned here. First by G. Stampacchia [68] who introduced 
the so-called duality solutions, then L. Boccardo and T. Gallouet [8] who defined approximation solutions and F. Murat [61] who proposed to study the entropy solutions, and others [50,22]. All of these concepts ensure the existence and uniqueness of the solutions for to the Dirichlet problem. However, these ideas do not apply if one wants to investigate the usual solutions, in the sense of Schwartz distributions. Precise conditions for the existence and uniqueness of the distributional solutions (in terms of the ellipticity constant $K$ ) depend on the norms of the Hilbert transform in $\mathbb{R}^{n}$. That is why it is impossible to give definite answers at present. However, we are able to give here at least good bounds for $K$ to ensure those properties. Critical to our approach is the introduction of the so-called grand $L^{s}$-space:

$$
L^{s}\left(\Omega, \mathbb{R}^{n}\right) \subset \bigcap_{0<\varepsilon \leqslant s-1} L^{s-\varepsilon}\left(\Omega, \mathbb{R}^{n}\right), \quad s=\frac{n}{n-1},
$$

which we supply with the norm

$$
\|F\|_{s}=\sup _{0<\varepsilon \leqslant 1}\left(\varepsilon f|F|_{\Omega}^{\frac{n-\varepsilon}{n-1}}\right)^{\frac{n-1}{n-\varepsilon}}<\infty .
$$

Every measure $v$ can be written as $v=\operatorname{div} F$, with some $F \in L^{s}\left(\Omega, \mathbb{R}^{n}\right)$ and $s=$ $n /(n-1)$. Explicitely, we can write

$$
F(x)=\int_{\Omega} \nabla_{x} G(x, y) d v(y),
$$

where $G$ is the Green's function for the Laplacian on $\Omega$. Theorem 3 is a consequence of (1.22) applied to $r=(n-\varepsilon) /(n-1)$.

We close this section with similar questions for the nonlinear $p$-harmonic type equation

$$
\operatorname{div} A(x, \nabla u)=v=\operatorname{div} F,
$$

where $K^{-1}|\xi|^{p} \leqslant\langle A(x, \xi), \xi\rangle \leqslant K|\xi|^{p}$. The uniqueness problem for distrbutional solutions is far more complicated. What we need first of all are the estimates of the form

$$
\int_{\Omega}|\nabla u|^{\frac{(p-1)(n-\varepsilon)}{n-1}} \leqslant C(n, p) \int_{\Omega}|F|^{\frac{n-\varepsilon}{n-1}}
$$

for at least some small positive numbers $\varepsilon$. These estimates are known only when $p=n$ [35], in which case the distributional solutions actually belong to the grand Sobolev space $W_{0}^{1, n)}(\Omega)$. The equations with measure in the right hand side have been subjected to a great deal of investigation by G. Dolzmann, N. Hungerbuehler and S. Müller, see [25] and more references given there. Finally, an approach to the nonlinear theory of harmonic fields and related PDEs of the Cauchy-Riemann type, referred to as Hodge systems, is presented in [46]. 


\section{Part Three:}

\section{Quasiharmonic Fields with Distortion in $\operatorname{Exp}\left(\mathbb{R}^{n}\right)$}

In the sections to follow, we will address the questions of regularity of quasiharmonic fields with unbounded distortion. The results have profound consequences for the elliptic PDEs with anisotropic degeneracy. We will continue this theme later in the paper. Since the $L^{2}$-integrability fails when $\mathrm{e}^{K}$ is not locally integrable, we must assume that

$$
\mathrm{e}^{\gamma K} \in L_{l o c}^{1}(\Omega)
$$

for some $\gamma \geqslant 1$. To get the higher integrability theory off the ground we will actually assume that $\gamma=\gamma(n)$ is sufficiently large. The theory of BMO-functions and some Orlicz spaces are a major prerequisite which we must outline here for completeness of the arguments in the sequel.

\section{Majorization and commutators with BMO-functions}

A function $K: \Omega \rightarrow \mathbb{R}_{+}$on a measurable set $\Omega \subset \mathbb{R}^{n}$ is said to be BMO-majorized if there exists $K^{\prime} \in \operatorname{BMO}\left(\mathbb{R}^{n}\right)$ such that $K(x) \leqslant K^{\prime}(x)$ for a.e. $x \in \Omega$.

PROPOSITION 16.1. - A necessary and sufficient condition that $K=K(x)$ should be $B M O$-majorized in $\Omega$ is that

$$
\int_{\Omega} \frac{\mathrm{e}^{\gamma K(x)} d x}{1+|x|^{n+1}}<\infty
$$

for some positive number $\gamma$.

The proof is based on a result of R. Coifman and R. Rochberg [18], which we state as follows.

LEMmA 16.2. - Let $\mathbf{M} \mu$ denote the Hardy-Littlewood maximal function of a Radon measure $\mu$ in $\mathbb{R}^{n}$, and suppose that $\mathbf{M} \mu(x)$ is finite and positive at some point $x$. Then

$$
\|\log (\mathbf{M} \mu)\|_{\mathrm{BMO}\left(\mathbb{R}^{n}\right)} \leqslant C(n) .
$$

Proof of Proposition 16.1. - Suppose (16.1) holds. We need only consider $\Omega=\mathbb{R}^{n}$, since otherwise we look at $K$ as equal to zero outside $\Omega$. The majorizing function in question can be defined as

$$
K^{\prime}(x)=\frac{1}{\gamma} \log \mathbf{M}\left(\frac{\mathrm{e}^{\gamma K(x)}}{1+|x|^{n+1}}\right)+\frac{1}{\gamma} \log \left(1+|x|^{n+1}\right) \geqslant K(x) .
$$

It is clear that $\left\|K^{\prime}\right\|_{\mathrm{BMO}} \leqslant C(n) / \gamma$.

For the converse, suppose that $K(x) \leqslant K^{\prime}(x)$ a.e. in $\Omega$ with $K^{\prime} \in \mathrm{BMO}\left(\mathbb{R}^{n}\right)$. Global integrability properties of BMO-functions follow from John-Nirenberg lemma. Among 
such properties is the following inequality

$$
\int_{\mathbb{R}^{n}} \frac{\mathrm{e}^{\gamma\left|K^{\prime}(x)-K_{0}^{\prime}\right|}}{1+|x|^{n+1}} d x \leqslant A(n),
$$

where $\gamma=\frac{C(n)}{\left\|K^{\prime}\right\|_{\text {BMO }}}$ and $K_{0}^{\prime}$ stands for the $L^{1}$-mean of $K^{\prime}$ over the unit cube. This completes the proof of Proposition 16.1.

Neither the hypotheses nor the forthcoming conclusions will be affected if we replace $K$ by $K^{\prime}$. Instead of introducing new symbols, we simply assume that the original distortion function satisfies

$$
\|K\|_{\mathrm{BMO}\left(\mathbb{R}^{n}\right)} \leqslant \frac{C(n)}{\gamma} .
$$

It will be convenient to introduce the weighted $L^{2}$-space of vector fields in $\mathbb{R}^{n}$

$$
L^{2}\left(\mathbb{R}^{n}, d \omega\right) \subset L^{2}\left(\mathbb{R}^{n}, d x\right),
$$

where $d \omega(x)=K^{2}(x) d x$. We realize that the Hilbert transform

$$
\mathbf{S}: L^{2}\left(\mathbb{R}^{n}, d \omega\right) \rightarrow L^{2}\left(\mathbb{R}^{n}, d \omega\right)
$$

is still bounded. In view of (16.2) its norm depends only on the dimension, but not on $K$. Indeed, for $F \in L^{2}\left(\mathbb{R}^{n}, d \omega\right)$ we have

$$
\begin{aligned}
\|\mathbf{S} F\|_{L^{2}\left(\mathbb{R}^{n}, d \omega\right)} & =\|K \mathbf{S} F\|_{2} \\
& \leqslant\|\mathbf{S}(K F)\|_{2}+\|(K \mathbf{S}-\mathbf{S} K) F\|_{2} \\
& \leqslant\|K F\|_{2}+C(n)\|K\|_{\mathrm{BMO}}\|F\|_{2} \\
& \leqslant C(n)\|F\|_{L^{2}\left(\mathbb{R}^{n}, d \omega\right) .}
\end{aligned}
$$

Here we have used an estimate for the commutator $K \mathbf{S}-\mathbf{S} K$ of $\mathbf{S}$ with the operator of multiplication by $K$. Such estimates will be frequently employed in the sequel, thus we state them as a lemma.

LEMmA 16.3. - Let $\mathbf{S}$ be a singular integral operator in $\mathbb{R}^{n}$ and let $K \in \mathrm{BMO}\left(\mathbb{R}^{n}\right)$. Then $K \mathbf{S}-\mathbf{S} K: L^{p}\left(\mathbb{R}^{n}, d x\right) \rightarrow L^{p}\left(\mathbb{R}^{n}, d x\right), 1<p<\infty$, has norm bounded by $C_{p}(n)\|K\|_{\text {BMO. }}$

This elegant result belongs to R. Coifman, R. Rochberg and G. Weiss [19]. We shall also discuss it in the context of Orlicz spaces later on.

\section{The $L^{2}$-inverse of $\mathbf{I}-\mu \mathbf{S}$}

With the above preliminaries we can now establish the following invertibility result

THEOREM 17.1. - There is a bounded linear operator $\Pi: L^{2}\left(\mathbb{R}^{n}, d \omega\right) \rightarrow L^{2}\left(\mathbb{R}^{n}\right.$, $d x)$ such that

$$
(\mathbf{I}-\mu \mathbf{S}) \circ \boldsymbol{\Pi}=\boldsymbol{\Pi} \circ(\mathbf{I}-\mu \mathbf{S})=\mathbf{I}: L^{2}\left(\mathbb{R}^{n}, d \omega\right) \rightarrow L^{2}\left(\mathbb{R}^{n}, d x\right) .
$$


Precisely, we have

$$
\|\Pi G\|_{L^{2}\left(\mathbb{R}^{n}, d x\right)} \leqslant 2\|G\|_{L^{2}\left(\mathbb{R}^{n}, d \omega\right)} .
$$

Proof. - We begin with a technical but very useful point-wise inequality

$$
|\mathbf{S} F|^{2}+|F|^{2} \leqslant 2 K\left(|\mathbf{S} F|^{2}-|F|^{2}\right)+4 K^{2}|(\mathbf{I}-\mu \mathbf{S}) F|^{2} .
$$

To see this, we write

$$
|(\mathbf{I}-\mu \mathbf{S}) F| \geqslant|F|-\|\mu\||\mathbf{S} F| \geqslant|F|-\frac{K-1}{K+1}|\mathbf{S} F|
$$

or, equivalently

$$
|\mathbf{S} F|+|F| \leqslant K(|\mathbf{S} F|-|F|)+(K+1)|F-\mu \mathbf{S} F| .
$$

Multiplying both sides by $2(|\mathbf{S} F|+|F|)$ gives

$$
\begin{aligned}
2(|\mathbf{S} F|+|F|)^{2} & \leqslant 2 K\left(|\mathbf{S} F|^{2}-|F|^{2}\right)+2(K+1)|F-\mu \mathbf{S} F|(|\mathbf{S} F|+|F|) \\
& \leqslant 2 K\left(|\mathbf{S} F|^{2}-|F|^{2}\right)+(K+1)^{2}|F-\mu \mathbf{S} F|^{2}+(|\mathbf{S} F|+|F|)^{2} .
\end{aligned}
$$

Inequality (17.3) is now straightforward.

We shall establish the existence of the operator $\Pi$ by proving the following estimate

$$
\|F\|_{L^{2}\left(\mathbb{R}^{n}, d x\right)} \leqslant 2\|(\mathbf{I}-\mu \mathbf{S}) F\|_{L^{2}\left(\mathbb{R}^{n}, d \omega\right)}
$$

for $F \in L^{2}\left(\mathbb{R}^{n}, d x\right)$. It is, therefore, natural to try to integrate (17.3). Some technical difficulties arise because the product $K\left(|\mathbf{S} F|^{2}-|F|^{2}\right)$ need not be integrable. For this reason, prior to integration, we divide (17.3) by $1+\varepsilon K(x)$ reducing the inequality to

$$
\frac{|\mathbf{S} F|^{2}+|F|^{2}}{1+\varepsilon K} \leqslant 2 k\left(|\mathbf{S} F|^{2}-|F|^{2}\right)+4 K^{2}|F-\mu \mathbf{S} F|^{2} .
$$

Here $\varepsilon>0$ and we notice that the new factor $k=K /(1+\varepsilon K)$ is bounded. At the end we will let parameter $\varepsilon$ go to zero. It is worth pointing out that the BMO-norm of $k$ does not depend on $\varepsilon$

$$
\|k\|_{\mathrm{BMO}} \leqslant 2\|K\|_{\mathrm{BMO}} \leqslant \frac{2 c(n)}{\gamma}
$$

as it is easy to check.

Next, with the aid of Hodge decomposition we can write $F=E-B$, where $B \in \mathcal{B}^{2}\left(\mathbb{R}^{n}\right)$ and $E \in \mathcal{E}^{2}\left(\mathbb{R}^{n}\right),\|F\|_{2}^{2}=\|B\|_{2}^{2}+\|E\|_{2}^{2}$. By the definition of the Hilbert transform $\mathbf{S} F=E+B$ and $|\mathbf{S} F|^{2}-|F|^{2}=4\langle B, E\rangle$. Then, integrating (17.5) yields

$$
\int_{\mathbb{R}^{n}} \frac{|B|^{2}+|E|^{2}}{1+\varepsilon K} \leqslant 4 \int_{\mathbb{R}^{n}}\langle k B, E\rangle+2\|F-\mu \mathbf{S} F\|_{L^{2}\left(\mathbb{R}^{n}, d \omega\right)}^{2} .
$$


To effectively estimate $\langle k B, E\rangle$ we apply Hodge decomposition of the vector field $k B \in L^{2}\left(\mathbb{R}^{n}, d x\right)$, that is

$$
k B=B^{\prime}+E^{\prime} .
$$

Here we can use the projection operators to compute the components $B^{\prime}$ and $E^{\prime}$, namely

$$
B^{\prime}=\mathbf{B}(k B) \in \mathcal{B}^{2}\left(\mathbb{R}^{n}\right) \quad \text { and } \quad E^{\prime}=\mathbf{E}(k B) \in \mathcal{E}^{2}\left(\mathbb{R}^{n}\right) .
$$

Since the vector fields $B^{\prime}$ and $E$ are orthogonal in $L^{2}\left(\mathbb{R}^{n}, \mathbb{R}^{n}\right)$ we are reduced to estimating the integral of $\left\langle E^{\prime}, E\right\rangle$. By Hölder's inequality

$$
\left|\int_{\mathbb{R}^{n}}\left\langle E^{\prime}, E\right\rangle\right| \leqslant\left\|E^{\prime}\right\|_{2}\|E\|_{2}
$$

The necessary bound for $\left\|E^{\prime}\right\|_{2}$ will follow almost immediately once we express $E^{\prime}$ in the form of a commutator,

$$
E^{\prime}=(\mathbf{E} k-k \mathbf{E}) B .
$$

This is legitimate because the operator $\mathbf{E}$ vanishes on divergence free vector fields. Now Lemma 16.3 yields

$$
\left\|E^{\prime}\right\|_{2} \leqslant C(n)\|k\|_{\mathrm{BMO}}\|B\|_{2} \leqslant \frac{C(n)}{\gamma}\|B\|_{2} .
$$

Therefore,

$$
\left|\int_{\mathbb{R}^{n}}\langle k B, E\rangle\right| \leqslant\left\|E^{\prime}\right\|_{2}\|E\|_{2} \leqslant \frac{C(n)}{\gamma} \int_{\mathbb{R}^{n}}\left(|B|^{2}+|E|^{2}\right) .
$$

On substituting it into (17.7), we obtain

$$
\int_{\mathbb{R}^{n}} \frac{|B|^{2}+|E|^{2}}{1+\varepsilon K} \leqslant \frac{4 C(n)}{\gamma} \int_{\mathbb{R}^{n}}\left(|B|^{2}+|E|^{2}\right)+2\|F-\mu \mathbf{S} F\|_{L^{2}\left(\mathbb{R}^{n}, d \omega\right)}^{2}
$$

Monotone Convergence Theorem yields the same inequality with $\varepsilon=0$. At this point we shall choose $\gamma$ sufficiently large, to ensure that the integral in the right hand side is absorbed by the left hand side. It results in the inequality:

$$
\frac{1}{2} \int_{\mathbb{R}^{n}}|F|^{2}=\frac{1}{2} \int_{\mathbb{R}^{n}}\left(|B|^{2}+|E|^{2}\right) \leqslant 2\|F-\mu \mathbf{S} F\|_{L^{2}\left(\mathbb{R}^{n}, d \omega\right)}^{2}
$$

which is the same as (17.4).

To settle matters finally, we need to solve the equation

$$
(\mathbf{I}-\mu \mathbf{S}) F=G
$$


for $F \in L^{2}\left(\mathbb{R}^{n}, d x\right)$, where $G$ is a given vector field in $L^{2}\left(\mathbb{R}^{n}, d \omega\right)$. Uniqueness certainly follows from (17.4). The only point remaining concerns the existence of the solution. To this effect, we approximate $\mu$ by

$$
\mu_{m}(x)= \begin{cases}\mu(x) & \text { if }\|\mu(x)\| \leqslant \frac{m-1}{m}, \\ \frac{(m-1) \mu(x)}{m\|\mu(x)\|} & \text { otherwise, }\end{cases}
$$

where $m=1,2, \ldots$ Note that $\left\|\mu_{m}(x)\right\| \leqslant 1-\frac{1}{m}$. It is also important to pay attention on the following bound independent of $m$

$$
\left\|\mu_{m}(x)\right\| \leqslant \frac{K(x)-1}{K(x)+1}, \quad m=1,2, \ldots
$$

Since the operator $\mathbf{I}-\mu_{m} \mathbf{S}$ is invertible in $L^{2}\left(\mathbb{R}^{n}, d x\right)$ we can solve the Beltrami equation

$$
F_{m}-\mu_{m} \mathbf{S} F_{m}=G \in L^{2}\left(\mathbb{R}^{n}, d \omega\right) \subset L^{2}\left(\mathbb{R}^{n}, d x\right)
$$

for $F_{m} \in L^{2}\left(\mathbb{R}^{n}, d x\right)$. On account of (17.4) the following uniform $L^{2}$-bounds hold

$$
\left\|F_{m}\right\|_{L^{2}\left(\mathbb{R}^{n}, d x\right)} \leqslant 2\|G\|_{L^{2}\left(\mathbb{R}^{n}, d \omega\right)} .
$$

We may certainly assume that $\left\{F_{m}\right\}$ converges weakly to an $F \in L^{2}\left(\mathbb{R}^{n}, d x\right)$, if not, we consider a subsequence. It follows from the boundedness of $\mathbf{S}$ that $\mathbf{S} F_{m}$ are converging to $\mathbf{S} F$, weakly in $L^{2}\left(\mathbb{R}^{n}, d x\right)$. Note that $\mu_{m}(x) \rightarrow \mu(x)$ and $\left\|\mu_{m}(x)\right\| \leqslant 1$, at almost every $x \in \mathbb{R}^{n}$. This implies weak convergence of $F_{m}-\mu_{m} \mathbf{S} F_{m}$ to $F-\mu \mathbf{S} F$, proving that $F$ is a solution of Eq. (17.10). Lastly, we define $\Pi G$ to be this solution $F \in L^{2}\left(\mathbb{R}^{n}, d x\right)$, completing the proof of Theorem 17.1.

Our ultimate goal will be to extend the operator $\Pi: L^{2}\left(\mathbb{R}^{n}, d \omega\right) \rightarrow L^{2}\left(\mathbb{R}^{n}, d x\right)$ to the Zygmund classes $L^{2} \log ^{\alpha} L$. But first we have to establish somewhat cumbersome technical details associated with the Orlicz spaces.

\section{Some Orlicz spaces}

A continuous and increasing function $\Phi:[0, \infty) \rightarrow[0, \infty)$, with $\Phi(0)=0$ and $\lim _{t \mapsto \infty} \Phi(t)=\infty$, will be called an Orlicz function. Let $(\Omega, \omega)$ be a $\sigma$-finite measure space and $W$ a finite dimensional normed space. The generalized Orlicz class $L^{\Phi}(\Omega, W)$ consists of all measurable functions $f: \Omega \rightarrow W$ such that

$$
\|f\|_{\Phi}=\inf \left\{\lambda>0 ; \int_{\Omega} \Phi\left(\frac{|f|}{\lambda}\right) \leqslant 1\right\} .
$$

$L^{\Phi}(\Omega, W)$ is a complete linear metric space [63]. As a note of warning, \|\|$_{\Phi}$ is not a norm. This nonlinear functional, also known as Luxemburg functional, is homogeneous 
but it fails to satisfy the triangle inequality when $\Phi$ is not convex. In the convex case, \|\|$_{\Phi}$ is a norm and $L^{\Phi}(\Omega, W)$ becomes a Banach space.

Sometimes, to signify the dependence on the measure $d \omega$, we use the notation $L^{\Phi}(\Omega, d \omega)$ in which case the target space $W$ will be recognized from the context.

The Zygmund classes $L^{p} \log ^{\alpha} L$, corresponding to $\Phi(t)=t^{p} \log ^{\alpha}(\mathrm{e}+t), 1 \leqslant p<$ $\infty, \alpha \in \mathbb{R}$, will be of particular interest to us. An elementary calculation shows that these Orlicz functions are increasing and convex, whenever $\alpha \geqslant 1-p$. The following estimates are straightforward

$$
\begin{aligned}
\|f\|_{L^{p} \log ^{-1} L} & \leqslant\|f\|_{p} \leqslant\|f\|_{L^{p} \log L} \\
& \leqslant\left[\int|f|^{p} \log \left(\mathrm{e}+\frac{|f|}{\|f\|_{p}}\right)\right]^{1 / p} \leqslant 2\|f\|_{L^{p} \log L} .
\end{aligned}
$$

The integral expression in the middle defines a norm in $L^{p} \log L$, which is equivalent to the Luxemburg norm; the triangle inequality is far from being obvious. We have the following Hölder type inequalities for real-valued functions

$$
\|A B\|_{L^{c} \log ^{\gamma} L} \leqslant C_{\alpha \beta}(a, b)\|A\|_{L^{a} \log ^{\alpha} L}\|B\|_{L^{b} \log ^{\beta} L},
$$

where the exponents $a, b>1$ and $\alpha, \beta \in \mathbb{R}$ are coupled by the equations

$$
\frac{1}{c}=\frac{1}{a}+\frac{1}{b}, \quad \frac{\gamma}{c}=\frac{\alpha}{a}+\frac{\beta}{b} .
$$

Thus, in particular

$$
\|f g\|_{1} \leqslant c\|f\|_{L^{2} \log ^{-\alpha} L}\|g\|_{L^{2} \log ^{\alpha} L}
$$

and

$$
\|f g\|_{L \log L} \leqslant c\|f\|_{L^{2} \log L}\|g\|_{L^{2} \log L} .
$$

If $W$ is an inner product space, then the nondegenerate bilinear form $\int\langle f, g\rangle$ gives rise to the duality between $L^{2} \log ^{-\alpha} L$ and $L^{2} \log ^{\alpha} L$. Here is another useful estimate

LEMMA 18.1. - For $f \in L^{2} \log L(\Omega, W)$, we have

$$
\left\|f \log \left(\mathrm{e}+\frac{|f|}{\|f\|_{2}}\right)\right\|_{L^{2} \log ^{-1} L} \leqslant 2\|f\|_{L^{2} \log L} .
$$

Proof. - To shorten notation, we recall the Orlicz spaces (1.8) corresponding to $P(t)=t^{2} \log (\mathrm{e}+t)$ and $Q(t)=t^{2} \log ^{-1}(\mathrm{e}+t)$. It follows from the definition of the Luxemburg norm that

$$
\begin{aligned}
1 & =\int \frac{|f|^{2}}{\|f\|_{P}^{2}} \log \left(\mathrm{e}+\frac{|f|}{\|f\|_{P}}\right) \\
& \leqslant \int \frac{|f|^{2} \log ^{2}\left(\mathrm{e}+\frac{|f|}{\|f\|_{P}}\right)}{\|f\|_{P}^{2} \log \left[\mathrm{e}+\frac{|f|}{\|f\|_{P}} \log \left(\mathrm{e}+\frac{|f|}{\|f\|_{P}}\right)\right]} \\
& =\int Q\left(\frac{f}{\|f\|_{P}} \log \left[\mathrm{e}+\frac{|f|}{\|f\|_{P}}\right]\right) .
\end{aligned}
$$


Hence, by the definition of the $Q$-norm

$$
\left\|f \log \left(\mathrm{e}+\frac{|f|}{\|f\|_{P}}\right)\right\|_{Q} \leqslant\|f\|_{P} .
$$

We only need to sharpen the left hand side of this inequality in such a way that $\|f\|_{2}$ will take place of $\|f\|_{P}$. This goes as follows

$$
\begin{aligned}
\left\|f \log \left(\mathrm{e}+\frac{|f|}{\|f\|_{2}}\right)\right\|_{Q} & \leqslant\left\|f \log \left(\mathrm{e}+\frac{|f|}{\|f\|_{P}}\right)\right\|_{Q}+\left\|f \log \left(1+\frac{\|f\|_{P}}{\|f\|_{2}}\right)\right\| \\
& \leqslant\|f\|_{P}+\|f\|_{Q} \log \left(1+\frac{\|f\|_{P}}{\|f\|_{2}}\right) \\
& \leqslant\|f\|_{P}+\|f\|_{Q} \frac{\|f\|_{P}}{\|f\|_{2}}=2\|f\|_{P},
\end{aligned}
$$

completing the proof of the lemma.

The following Orlicz space extension of the Marcinkiewicz interpolation theorem will be useful.

LEMMA 18.2. - Let $\mathbf{M}$ be a sub-additive and homogeneous operator from $L^{q}(\Omega, W)$ $+L^{p}(\Omega, W), 0<q<p<\infty$, to the space of measurable functions on $\Omega$, that is

$$
\left\{\begin{array}{l}
|\mathbf{M}(f+g)| \leqslant|\mathbf{M} f|+|\mathbf{M} g| \\
\mathbf{M}(\alpha f)=\alpha \mathbf{M}(f), \quad \alpha \geqslant 0
\end{array}\right.
$$

Suppose that $\mathbf{M}$ is simultaneously of weak type $(q, q)$ and weak-type $(p, p)$. That is, for each $\alpha>0$, we have

$$
\mid\{x:|\mathbf{M} f(x)|>\alpha\} \leqslant \begin{cases}\left(\frac{A_{q}\|f\|_{q}}{\alpha}\right)^{q} & \text { for } f \in L^{q}(\Omega, W), \\ \left(\frac{A_{p}\|f\|_{p}}{\alpha}\right)^{p} & \text { for } f \in L^{p}(\Omega, W) .\end{cases}
$$

Let $\Phi$ be an Orlicz function such that $t^{a} \prec \Phi \prec t^{b}$, for some exponents $q<a<b<p$. This simply means that the function $t^{-a} \Phi(t)$ is increasing, while $t^{-b} \Phi(t)$ is decreasing.

Then $\mathbf{M}$ is a bounded operator from $L^{\Phi}(\Omega, W)$ into itself, and we have

$$
\|\mathbf{M} f\|_{\Phi} \leqslant C\|f\|_{\Phi} \quad \text { for } \in L^{\Phi}(\Omega, W) .
$$

The constant $C=C_{\Phi}$ is determined by the equation

$$
\frac{a}{a-q}\left(\frac{2 A_{q}}{C}\right)^{q}+\frac{b}{p-b}\left(\frac{2 A_{p}}{C}\right)^{p}=1 .
$$

Proof. - Because of homogeneity we may assume that $\|f\|_{\Phi}=1$, which gives

$$
\int_{\Omega} \Phi(|f|)=1 .
$$


It also involves no loss of generality in assuming that the constant $C=C_{\Phi}$ at (18.10) equals 1. For, if not, we consider the operator $C^{-1} \mathbf{M}$ instead of $\mathbf{M}$. We then mimic the idea of Marcinkiewicz as presented in [69, pp. 21-22]. Let $\lambda(t)=\mid\{x ;|\mathbf{M} f(x)|>$ $t\} \mid$ denote the distribution function of $\mathbf{M} f$. A slight modification of the proof of inequality (19) in $[69$, p. 22] actually shows that

$$
\lambda(t) \leqslant\left(\frac{2 A_{q}}{t}\right)^{q} \int_{|f|>t}|f|^{q}+\left(\frac{2 A_{p}}{t}\right)^{p} \int_{|f| \leqslant t}|f|^{p} .
$$

Next, we express $\int \Phi(|\mathbf{M} f|)$ by means of the Riemann-Stieltjes integral with respect to $\Phi(t)$ and use (18.12) to obtain

$$
\begin{aligned}
\int_{\Omega} \Phi(|\mathbf{M} f|) & =\int_{0}^{\infty} \lambda(t) d \Phi(t) \\
& \leqslant\left(2 A_{q}\right)^{q} \int_{0}^{\infty}\left(\int_{|f|>t}|f|^{q}\right) \frac{d \Phi}{t^{q}}+\left(2 A_{p}\right)^{p} \int_{0}^{\infty}\left(\int_{|f| \leqslant t}|f|^{p}\right) \frac{d \Phi}{t^{p}} .
\end{aligned}
$$

Further, changing order of integration this reads as

$$
\int_{\Omega} \Phi(|\mathbf{M} f|) \leqslant\left(2 A_{q}\right)^{q} \int_{\Omega}|f|^{q}\left(\int_{0}^{|f|} \frac{d \Phi}{t^{q}}\right)+\left(2 A_{p}\right)^{p} \int_{\Omega}|f|^{p}\left(\int_{|f|}^{\infty} \frac{d \Phi}{t^{p}}\right) .
$$

We shall now estimate the line integrals. Integrating by parts, and with the aid of the hypothesis that $t^{a} \prec \Phi(t) \prec t^{b}$, we obtain

$$
\begin{aligned}
\int_{0}^{|f|} \frac{d \Phi}{t^{q}} & =\frac{\Phi(|f|)}{|f|^{q}}+q \int_{0}^{|f|} \frac{\Phi(t) d t}{t^{1+q}} \\
& \leqslant \frac{\Phi(|f|)}{|f|^{q}}+q \frac{\Phi(|f|)}{|f|^{a}} \int_{0}^{|f|} \frac{d t}{t^{1+q-a}}=\frac{a}{a-q} \frac{\Phi(|f|)}{|f|^{q}} .
\end{aligned}
$$

In much the same way we get the inequality

$$
\int_{|f|}^{\infty} \frac{d \Phi}{t^{p}} \leqslant \frac{b}{p-b} \frac{\Phi(|f|)}{|f|^{p}}
$$

Finally, we arrive at the estimate

$$
\int_{\Omega} \Phi(|\mathbf{M} f|) \leqslant\left[\frac{a}{a-q}\left(2 A_{q}\right)^{q}+\frac{b}{p-b}\left(2 A_{p}\right)^{p}\right] \int_{\Omega} \Phi(|f|)=1 .
$$


Here, by virtue of the normalization of the operator $\mathbf{M}$, the constant in the square brackets equals 1 . Now the definition of Luxemburg's norm implies

$$
\|\mathbf{M} f\|_{\Phi} \leqslant 1=C_{\Phi}\|f\|_{\Phi}
$$

completing the proof of the lemma.

A few words should be said about this result. While the interpolation theory has long been discussed in the context of Orlicz spaces [63,36,52], the details like in Lemma 18.2 are rather new, and much unfamiliar. Also, we have found it desirable to include the proof of (18.9) as it is easier than to combine the existing more general results scattered throughout the research journals.

Lemma 18.2 furnishes a convenient tool for establishing boundedness of singular integrals, maximal operators and some commutators.

Lemma 18.3. - Let $\Phi(t)=t^{p} \log ^{\alpha}(\mathrm{e}+t), 1<p<\infty, \alpha \in \mathbb{R}$, and let $\mathbf{T}$ be a singular integral operator in $\mathbb{R}^{n}$. Then

$$
\|\mathbf{T}(f)\|_{L^{p} \log ^{\alpha} L} \leqslant C_{p}(\alpha)\|f\|_{L^{p} \log ^{\alpha} L} .
$$

The same holds for the Hardy-Littlewood maximal function

$$
\mathbf{M} f(x)=\sup \left\{f_{Q}|f(y)| d y ; x \in Q \subset \mathbb{R}^{n}\right\}
$$

supremum being taken with respect to all cubes containing $x \in \mathbb{R}^{n}$.

$$
\|\mathbf{M} f\|_{L^{p} \log ^{\alpha} L} \leqslant c_{p}(n, \alpha)\|f\|_{L^{p} \log ^{\alpha} L} .
$$

The Orlicz space extension of Lemma 16.3 reads as

$$
\|(k \mathbf{T}-\mathbf{T} k) F\|_{L^{p} \log ^{\alpha} L} \leqslant c_{p}(n, \alpha)\|k\|_{\mathrm{BMO}}\|F\|_{L^{p} \log ^{\alpha} L} .
$$

Let us take a little time now to analyze more closely the proof of Theorem 17.1. Although we did not emphasize it there the idea behind the calculation was that the div-curl product $\langle B, E\rangle$ belongs to the Hardy space $\mathcal{H}^{1}\left(\mathbb{R}^{n}\right)$, see [17]. The key estimate at (17.9) can actually be derived from the duality of $\mathcal{H}^{1}$ and BMO

$$
\left|\int_{\mathbb{R}^{n}} k\langle B, E\rangle\right| \leqslant\|k\|_{\mathrm{BMO}}\|\langle B, E\rangle\|_{\mathcal{H}^{1}} \leqslant C_{n}\|k\|_{\mathrm{BMO}}\|B\|_{2}\|E\|_{2} .
$$

In the context of Orlicz norms, this fact reads as follows:

LeMma 18.4. - Let $[B, E]$ be a div-curl couple with $B \in L^{2} \log ^{\alpha} L\left(\mathbb{R}^{n}, \mathbb{R}^{n}\right)$ and $E \in L^{2} \log ^{-\alpha} L\left(\mathbb{R}^{n}, \mathbb{R}^{n}\right), \alpha \in \mathbb{R}$ and $k \in L^{\infty}\left(\mathbb{R}^{n}\right)$. Then

$$
\left|\int_{\mathbb{R}^{n}} k\langle B, E\rangle\right| \leqslant C(n, \alpha)\|k\|_{\mathrm{BMO}}\|B\|_{L^{2} \log ^{\alpha} L}\|E\|_{L^{2} \log ^{-\alpha} L} .
$$


Proof. - The arguments are much the same as those in the proof of Theorem 17.1, the only difference is that we apply Hodge decomposition to the vector fields in $L^{2} \log ^{\alpha} L\left(\mathbb{R}^{n}, d x\right)$. Here are some details

$$
k B=B^{\prime}+E^{\prime}=\mathbf{B}(k B)+\mathbf{E}(k B) .
$$

The Orlicz space version of identity (2.6) shows that the integral of $\left\langle B^{\prime}, E\right\rangle$ vanishes. Thus we obtain

$$
\left|\int_{\mathbb{R}^{n}} k\langle B, E\rangle\right|=\left|\int_{\mathbb{R}^{n}}\left\langle E^{\prime}, E\right\rangle\right| \leqslant C(n)\left\|E^{\prime}\right\|_{L^{2} \log ^{\alpha} L}\|E\|_{L^{2} \log ^{-\alpha} L} .
$$

Writing $E^{\prime}=(\mathbf{E} k-k \mathbf{E}) B$ and using Lemma 18.3, we conclude with the required estimate

$$
\left\|E^{\prime}\right\|_{L^{2} \log ^{\alpha} L} \leqslant C(n, \alpha)\|k\|_{\mathrm{BMO}}\|B\|_{L^{2} \log ^{\alpha} L}
$$

completing the proof of the lemma.

Inequality (18.18) will play crucial role in the extension of the operator $\Pi: L^{2}\left(\mathbb{R}^{n}, d \omega\right) \rightarrow L^{2}\left(\mathbb{R}^{n}, d x\right)$ to the Orlicz spaces $L^{2} \log ^{\alpha} L\left(\mathbb{R}^{n}, d \omega\right)$.

\section{A restriction at infinity}

Some global integrability properties of $\mathrm{e}^{\gamma K}$ are necessary for the invertibility of the operator $\mathbf{I}-\mu \mathbf{S}$ in spaces different from $L^{2}\left(\mathbb{R}^{n}, \mathbb{R}^{n}\right)$.

Recall that we always require $\mathrm{e}^{\gamma K} \in L_{l o c}^{1}\left(\mathbb{R}^{n}\right)$ for sufficiently large $\gamma=\gamma(n)$. Here we shall impose further restriction on $K$ at infinity, namely

$$
\mathrm{e}^{\gamma K}=f+b \in L^{1}\left(\mathbb{R}^{n}\right)+L^{\infty}\left(\mathbb{R}^{n}\right) .
$$

This condition, like (16.1), is also invariant under majorization by a BMO-function. For this purpose, we shall need to examine the function

$$
K^{\prime}=\frac{1}{\gamma} \log \left[\left(\mathbf{M}|f|^{1 / 2}\right)^{2}+\mathbf{M} b\right]
$$

where $\mathbf{M}$ stands for the Hardy-Littlewod maximal operator. It is evident that $K^{\prime}(x) \geqslant$ $K(x)$, everywhere. To estimate its BMO-norm the following elementary inequality will suit us nicely

$$
\|\log (X+Y)\|_{\mathrm{BMO}} \leqslant 3\|\log X\|_{\mathrm{BMO}}+3\|\log Y\|_{\mathrm{BMO}},
$$

where $X$ and $Y$ are arbitrary positive functions. The verification of (19.3) is left to the interested reader. Hence, by Lemma (16.2), we obtain

$$
\left\|K^{\prime}\right\|_{\text {ВMO }} \leqslant \frac{6}{\gamma}\left\|\log \left(\mathbf{M}|f|^{1 / 2}\right)\right\|_{\text {BMO }}+\frac{3}{\gamma}\|\log \mathbf{M} b\|_{\text {BMO }} \leqslant \frac{C(n)}{\gamma} .
$$


A decomposition similar to that of (19.1) is worth noting

$$
\mathrm{e}^{\gamma K^{\prime}}=f^{\prime}+b^{\prime}=\left(\mathbf{M}|f|^{1 / 2}\right)^{2}+\mathbf{M} b \in L^{1}\left(\mathbb{R}^{n}\right)+L^{\infty}\left(\mathbb{R}^{n}\right) .
$$

Moreover, for the new components $f^{\prime}=\left(\mathbf{M}|f|^{1 / 2}\right)^{2}$ and $b^{\prime}=\mathbf{M} b$ we have the following uniform bounds

$$
\begin{aligned}
& \left\|f^{\prime}\right\|_{1}=\left\|\mathbf{M}\left(|f|^{1 / 2}\right)\right\|_{2}^{2} \leqslant C(n)\|f\|_{1}, \\
& \left\|b^{\prime}\right\|_{\infty} \leqslant\|b\|_{\infty} .
\end{aligned}
$$

Next observe that for each cube $Q \subset \mathbb{R}^{n}$

$$
\mathrm{e}^{\gamma} \leqslant f_{Q} \mathrm{e}^{\gamma K} \leqslant f_{Q}|f|+f_{Q}|b| \leqslant \frac{\|f\|_{1}}{|Q|}+\inf _{Q} b^{\prime} .
$$

Expanding $Q$ to the entire space $\mathbb{R}^{n}$ we then conclude that $b^{\prime}(x) \geqslant \mathrm{e}^{\gamma}$, everywhere in $\mathbb{R}^{n}$. Thus $b^{\prime}=\mathrm{e}^{\gamma K_{0}}$, for some bounded function $1 \leqslant K_{0}(x) \leqslant \frac{1}{\gamma} \log \|b\|_{\infty}$. Decomposition (19.5) can be written as

$$
\mathrm{e}^{\gamma\left(K^{\prime}-K_{0}\right)}-1=\mathrm{e}^{-\gamma K_{0}}\left(\mathbf{M}|f|^{1 / 2}\right)^{2} \in L^{1}\left(\mathbb{R}^{n}\right)
$$

which shows, in particular, that $K_{0}(x) \leqslant K^{\prime}(x)$.

With these observations in mind, it is convenient (without loss of generality) to reformulate condition (19.1) as:

$$
\|K\|_{\mathrm{BMO}} \leqslant \frac{C(n)}{\gamma}
$$

and

$$
\mathrm{e}^{\gamma\left(K-K_{0}\right)}-1 \in L^{1}\left(\mathbb{R}^{n}\right)
$$

for some $K_{0} \in L^{\infty}\left(\mathbb{R}^{n}\right)$ such that $1 \leqslant K_{0}(x) \leqslant K(x)$. Furthermore, to accomodate explicit estimates the following quantity seems to be useful

$$
[K]=\left\|K_{0}\right\|_{\infty}+\frac{1}{\gamma} \int_{\mathbb{R}^{n}}\left[\mathrm{e}^{\gamma\left(K-K_{0}\right)}-1\right] .
$$

This quantity is controlled in terms of the original decomposition (19.1)

$$
[K] \leqslant \frac{C(n)}{\gamma}\left[\log \|b\|_{\infty}+\mathrm{e}^{-\gamma}\|f\|_{1}\right] .
$$

Another useful fact is that the norms $\|G\|_{L^{P}\left(\mathbb{R}^{n}, d \omega\right)}$ and $\|K G\|_{L^{P}\left(\mathbb{R}^{n}, d x\right)}$ are comparable, where $P(t)=t^{2} \log (\mathrm{e}+t)$. More precisely, for $\gamma \geqslant 10$, we have

$$
\begin{aligned}
\|G\|_{L^{P}\left(\mathbb{R}^{n}, d \omega\right)}^{2} & \leqslant\|K G\|_{L^{P}\left(\mathbb{R}^{n}, d x\right)}^{2} \\
& \leqslant 20\|G\|_{L^{P}\left(\mathbb{R}^{n}, d \omega\right)}^{2}+5[K]\|K G\|_{L^{2}\left(\mathbb{R}^{n}, d x\right)}^{2} \\
& \leqslant 25[K]\|G\|_{L^{P}\left(\mathbb{R}^{n}, d \omega\right)}^{2} .
\end{aligned}
$$


The first inequality follows from the definition of the Luxemburg norm but the second one needs some work. We begin by the following computation

$$
\begin{aligned}
\|K G\|_{L^{P}\left(\mathbb{R}^{n}, d x\right)}^{2} & \leqslant \int_{\mathbb{R}^{n}} K^{2}|G|^{2} \log \left(\mathrm{e}+\frac{K|G|}{\|K G\|_{2}}\right) \\
& \leqslant \int_{\mathbb{R}^{n}} K^{2}|G|^{2} \log \left(\mathrm{e}+\frac{|G|}{\|K G\|_{2}}\right)+\int_{\mathbb{R}^{n}} K^{3}|G|^{2} \\
& \leqslant 4\|G\|_{L^{P}\left(\mathbb{R}^{n}, d \omega\right)}^{2}+\int_{\mathbb{R}^{n}} K^{2}\left(K-K_{0}\right)|G|^{2}+\left\|K_{0}\right\|_{\infty}\|K G\|_{L^{2}\left(\mathbb{R}^{n}, d x\right)}^{2} .
\end{aligned}
$$

Next, we use an elementary inequality

$$
x y \leqslant \frac{x}{\gamma} \log \left(\mathrm{e}+\frac{x}{a}\right)+\frac{a}{\gamma}\left(\mathrm{e}^{\gamma y}-1\right),
$$

to find that

$$
\begin{aligned}
\int_{\mathbb{R}^{n}} K^{2}\left(K-K_{0}\right)|G|^{2} & \leqslant \frac{1}{\gamma} \int_{\mathbb{R}^{n}} K^{2}|G|^{2} \log \left(\mathrm{e}+\frac{K^{2}|G|^{2}}{\|K G\|_{2}^{2}}\right)+\frac{\|K G\|_{2}^{2}}{\gamma} \int_{\mathbb{R}^{n}}\left[\mathrm{e}^{\gamma\left(K-K_{0}\right)}-1\right] \\
& \leqslant \frac{8}{\gamma}\|K G\|_{L^{P}\left(\mathbb{R}^{n}, d x\right)}^{2}+\left([K]-\left\|K_{0}\right\|_{\infty}\right)\|K G\|_{2}^{2} .
\end{aligned}
$$

Combining all the above gives

$$
\|K G\|_{L^{P}\left(\mathbb{R}^{n}, d x\right)}^{2} \leqslant 4\|G\|_{L^{P}\left(\mathbb{R}^{n}, d \omega\right)}^{2}+[K]\|K G\|_{2}^{2}+\frac{4}{5}\|K G\|_{L^{P}\left(\mathbb{R}^{n}, d x\right)}^{2}
$$

from which (19.11) is immediate.

\section{Extension of $\Pi$ to $L^{2} \log L$}

Having disposed of all the necessary requisites, we consider the question whether the operator $\Pi$, originally defined in $L^{2}\left(\mathbb{R}^{n}, d \omega\right)$ with values in $L^{2}\left(\mathbb{R}^{n}, d x\right)$, extends to a continuous operator

$$
\Pi: L^{2} \log L\left(\mathbb{R}^{n}, d \omega\right) \rightarrow L^{2} \log L\left(\mathbb{R}^{n}, d x\right) .
$$

THEOREM 20.1. - Under the conditions on $K$ stated above, the operator $\Pi$ acts continuously from $L^{2} \log L\left(\mathbb{R}^{n}, d \omega\right)$ into $L^{2} \log L\left(\mathbb{R}^{n}, d x\right)$. It also verifies the following uniform bounds

$$
\begin{aligned}
\|\Pi G\|_{L^{2} \log L\left(\mathbb{R}^{n}, d x\right)} & \leqslant C(n)\|K G\|_{L^{2} \log L\left(\mathbb{R}^{n}, d x\right)}+C(n) \sqrt{[K]}\|K G\|_{L^{2}\left(\mathbb{R}^{n}, d x\right)} \\
& \leqslant C(n) \sqrt{[K]}\|G\|_{L^{2} \log L\left(\mathbb{R}^{n}, d \omega\right)} .
\end{aligned}
$$

Proof. - The extension will be established once we prove the estimate

$$
\|F\|_{P} \leqslant C(n)\|K(\mathbf{I}-\mu \mathbf{S}) F\|_{P}+C(n)[K]\|F\|_{2}
$$


for all $F \in L^{P}\left(\mathbb{R}^{n}, d x\right)=L^{2} \log L\left(\mathbb{R}^{n}, d x\right)$. We proceed analogously to the proof of inequality (17.4). Before making any calculation, however, we normalize $F$

$$
\|F\|_{2}=1 .
$$

Being homogeneous, inequality (20.3) is not affected by this assumption. Let $\mathbf{M} F$ denote the Hardy-Littlewood maximal function of $F$. This function is $L^{2}$-majorized by constants depending only on the dimension

$$
1 \leqslant\|\mathbf{M} F\|_{2} \leqslant C(n),
$$

by Maximal Theorem in $L^{2}\left(\mathbb{R}^{n}\right)$. Finally, we introduce an auxiliary function

$$
\lambda=\log (\mathrm{e}+\mathbf{M} F), \quad \text { thus }\|\lambda\|_{\mathrm{BMO}} \leqslant C(n)
$$

by (19.3) and Lemma 16.2.

Now, the pointwise inequality (17.3) is again our starting point. We multiply it by $\lambda$, divide by $1+\varepsilon K$ and integrate over $\mathbb{R}^{n}$, to obtain

$$
\int_{\mathbb{R}^{n}} \frac{|F|^{2} \log (\mathrm{e}+|F|)}{1+\varepsilon K} \leqslant 2 \int_{\mathbb{R}^{n}} k\left(|\mathbf{S} F|^{2}-|F|^{2}\right) \lambda+4 \int_{\mathbb{R}^{n}} K^{2}|F-\mu \mathbf{S} F|^{2} \lambda,
$$

where we recall that $k=\frac{K}{1+\varepsilon K} \in L^{\infty}\left(\mathbb{R}^{n}\right)$ and

$$
\|k\|_{\mathrm{BMO}} \leqslant \frac{C(n)}{\gamma} .
$$

Let us first take on the easier estimate of the second integral at the right hand side of (20.7). The claim is that

$$
\int_{\mathbb{R}^{n}} K^{2}|F-\mu \mathbf{S} F|^{2} \lambda \leqslant C(n)\|K(\mathbf{I}-\mu \mathbf{S}) F\|_{P}^{2}
$$

and follows from an elementary inequality $a^{2} \log (\mathrm{e}+b) \leqslant 2 a^{2} \log (\mathrm{e}+a)+b^{2}$, for nonnegative numbers. Indeed, let us denote

$$
G(x)=F(x)-\mu(x) \mathbf{S} F(x) .
$$

On substituting $a=\frac{K|G|}{\|K G\|_{P}}$ and $b=\mathbf{M} F$, we obtain

$$
\int_{\mathbb{R}^{n}} \lambda K^{2}|G|^{2} \leqslant 2 \int_{\mathbb{R}^{n}} K^{2}|G|^{2} \log \left(\mathrm{e}+\frac{K|G|}{\|K G\|_{P}}\right)+\|K G\|_{P}^{2}\|\mathbf{M} F\|_{2}^{2}
$$

The integral term in the right hand side equals $\|K G\|_{P}^{2}$, while the second term is no larger than $C(n)\|K G\|_{P}^{2}$, completing the proof of (20.9). 
We are left with the task of estimating the integral $\int_{\mathbb{R}^{n}} k\left(|\mathbf{S} F|^{2}-|F|^{2}\right) \lambda$. To this effect, we use div-curl decompositions $F=E-B$ and $\mathbf{S} F=E+B$, with components in the Orlicz classes. Namely, $B \in \mathcal{B}^{P}\left(\mathbb{R}^{n}\right)$ and $E \in \mathcal{E}^{P}\left(\mathbb{R}^{n}\right)$. Their $P$-norms are controlled by that of $F$

$$
\|B\|_{P}+\|E\|_{P} \leqslant C(n)\|F\|_{P} .
$$

Now, the integral in question takes the form

$$
\int_{\mathbb{R}^{n}} k\left(|\mathbf{S} F|^{2}-|F|^{2}\right) \lambda=4 \int_{\mathbb{R}_{n}} k\langle\lambda B, E\rangle .
$$

Although the vector field $\lambda B$ is no longer divergence free it, nevertheless, preserves important cancellation properties, since the factor $\lambda$ belongs to $\operatorname{BMO}\left(\mathbb{R}^{n}\right)$. To rigorously explain this phenomenon let us first show that $\lambda B \in L^{Q}\left(\mathbb{R}^{n}, d x\right)=L^{2} \log ^{-1} L\left(\mathbb{R}^{n}, d x\right)$. For this, we write

$$
\begin{aligned}
|\lambda B| & =|B| \log (\mathrm{e}+\mathbf{M} F) \leqslant(|F|+|\mathbf{S} F|) \log (\mathrm{e}+\mathbf{M} F) \\
& \leqslant(\mathbf{M} F+|\mathbf{S} F|) \log (\mathrm{e}+\mathbf{M} F+|\mathbf{S} F|) \\
& \leqslant(\mathbf{M} F+|\mathbf{S} F|) \log \left(\mathrm{e}+\frac{\mathbf{M} F+|\mathbf{S} F|}{\|\mathbf{M} F+|\mathbf{S} F|\|_{2}}\right)+C(n)(\mathbf{M} F+|\mathbf{S} F|) .
\end{aligned}
$$

In the latter step we have used the obvious inequality $\|\mathbf{M} F+\mathbf{S} F\|_{2} \leqslant A(n)\|F\|_{2}=$ $A(n)$. By Lemma 18.1 it follows that $\lambda B \in L^{Q}\left(\mathbb{R}^{n}, d x\right)$, and an estimate of its norm is also available.

$$
\begin{aligned}
\|\lambda B\|_{Q} & \leqslant 2\|\mathbf{M} F+|\mathbf{S} F|\|_{P}+C(n)\|\mathbf{M} F+|\mathbf{S} F|\|_{Q} \\
& \leqslant[2+C(n)]\|\mathbf{M} F+|\mathbf{S} F|\|_{P} .
\end{aligned}
$$

Since the maximal operator and the Hilbert transform are both bounded in $L^{P}\left(\mathbb{R}^{n}, d x\right)$, we conclude with the inequality

$$
\|\lambda B\|_{Q} \leqslant C(n)\|F\|_{P} .
$$

Now, to measure how close is $\lambda B$ to a divergence free vector field in the space $L^{Q}\left(\mathbb{R}^{n}, d x\right)$, we use Hodge decomposition

$$
\lambda B=B^{\prime}+E^{\prime},
$$

where $B^{\prime} \in \mathcal{B}^{Q}\left(\mathbb{R}^{n}\right)$ and $E^{\prime} \in \mathcal{E}^{Q}\left(\mathbb{R}^{n}\right)$. We then estimate the divergence free component by expressing it as $B^{\prime}=\mathbf{B}(\lambda B)$. Thus

$$
\left\|B^{\prime}\right\|_{Q}=C(n)\|\lambda B\|_{Q} \leqslant C(n)\|F\|_{P},
$$

since the projection operator $\mathbf{B}$ is bounded in $L^{Q}\left(\mathbb{R}^{n}, d x\right)$. Concerning the curl-free component $E^{\prime}$, we have better estimate due to the commutator representation

$$
E^{\prime}=\mathbf{E}(\lambda B)=(\mathbf{E} \lambda-\lambda \mathbf{E}) B,
$$


since the operator $\mathbf{E}$ vanishes on div-free fields. With the aid of Lemma 18.3 we see that $E^{\prime}$ belongs to the same space as the vector field $B$ does, which is $L^{P}\left(\mathbb{R}^{n}, d x\right)=$ $L^{2} \log L\left(\mathbb{R}^{n}, d x\right)$. Let us record two estimates. The first reads as

$$
\left\|E^{\prime}\right\|_{P} \leqslant C(n)\|\lambda\|_{\mathrm{BMO}}\|B\|_{P} \leqslant C(n)\|F\|_{P} .
$$

The second one (for use in the sequel) is the $L^{2}\left(\mathbb{R}^{n}, d x\right)$ - analogue of this,

$$
\left\|E^{\prime}\right\|_{2} \leqslant C(n)\|\lambda\|_{\mathrm{BMO}}\|B\|_{2} \leqslant C(n)\|F\|_{2}=C(n) .
$$

Returning to (20.11), we now split the integral of the right hand side in accordance with the decomposition of $\lambda B$ at (20.13)

$$
\int_{\mathbb{R}^{n}} k\left(|\mathbf{S} F|^{2}-|F|^{2}\right) \lambda=4 \int_{\mathbb{R}^{n}} k\left\langle B^{\prime}, E\right\rangle+4 \int_{\mathbb{R}^{n}} k\left\langle E^{\prime}, E\right\rangle .
$$

Lemma 18.4 gives a bound for the first integral in the right hand side

$$
\left|4 \int_{\mathbb{R}^{n}} k\left\langle B^{\prime}, E\right\rangle\right| \leqslant C(n)\|k\|_{\mathrm{BMO}}\left\|B^{\prime}\right\|_{Q}\|E\|_{P} \leqslant \frac{C(n)}{\gamma}\|F\|_{P}^{2}
$$

by (20.8), (20.14) and (20.10). What remains is to estimate the second integral in (20.18). It is at this point that we need the restrictions on $K$, stated in (19.7) and (19.8). Accordingly,

$$
\begin{aligned}
\left|\int_{\mathbb{R}^{n}} k\left\langle E^{\prime}, E\right\rangle\right| \leqslant & \left\|K_{0}\right\|_{\infty}\left\|E^{\prime}\right\|_{2}\|E\|_{2}+\int_{\mathbb{R}^{n}}\left(K-K_{0}\right)\left|E^{\prime}\right||E| \\
\leqslant & C(n)\left\|K_{0}\right\|_{\infty}\|F\|_{2}^{2}+\frac{1}{\gamma} \int_{\mathbb{R}^{n}}\left|E^{\prime}\right||E| \log \left(\mathrm{e}+\frac{\left|E^{\prime}\right||E|}{\left\|\left|E^{\prime}\right||E|\right\|_{1}}\right) \\
& +\frac{1}{\gamma}\left\|\left|E^{\prime}\right||E|\right\|_{1} \int_{\mathbb{R}^{n}}\left[\mathrm{e}^{\gamma\left(K-K_{0}\right)}-1\right] .
\end{aligned}
$$

Here we have applied the elementary inequality

$$
x y \leqslant \frac{x}{\gamma} \log \left(\mathrm{e}+\frac{x}{a}\right)+\frac{a}{\gamma}\left(\mathrm{e}^{\gamma y}-1\right)
$$

for positive numbers. The first integral is equivalent to the $L \log L$-norm of $\left|E^{\prime}\right||E|$, apply (18.2) with $p=1$. By inequality (18.5) and by $\left\|\left|E^{\prime}\right||E|\right\|_{1} \leqslant\left\|E^{\prime}\right\|_{2}\|E\|_{2} \leqslant$ $C(n)\|F\|_{2}^{2}$, we arrive at the estimate

$$
\begin{aligned}
\left|\int_{\mathbb{R}^{n}} k\left\langle E^{\prime}, E\right\rangle\right| & \leqslant C(n)[K]\|F\|_{2}^{2}+\frac{1}{\gamma}\left\|E^{\prime}\right\|_{P}\|E\|_{P} \\
& \leqslant C(n)[K]\|F\|_{2}^{2}+\frac{C(n)}{\gamma}\|F\|_{P}^{2}
\end{aligned}
$$

due to (20.16). 
Finally, combining (20.9), (20.18), (20.19) and (20.20) yields

$$
\int_{\mathbb{R}^{n}} \frac{|F|^{2} \log (\mathrm{e}+|F|)}{1+\varepsilon K} \leqslant \frac{C(n)}{\gamma}\|F\|_{P}^{2}+C(n)[K]\|F\|_{2}^{2}+C(n)\|K(\mathbf{I}-\mu \mathbf{S})\|_{P}^{2} .
$$

It is now legitimate to let $\varepsilon$ go to zero. In view of the normalization $\|F\|_{2}=1$, we are lead to the inequality claimed at (20.3). Indeed, by (18.2) it follows that

$$
\begin{aligned}
\|F\|_{P}^{2} & \leqslant \int_{\mathbb{R}^{n}}|F|^{2} \log \left(\mathrm{e}+\frac{|F|}{\|F\|_{2}}\right) \\
& \leqslant \frac{C(n)}{\gamma}\|F\|_{P}^{2}+C(n)[K]\|F\|_{2}^{2}+C(n)\|K(\mathbf{I}-\mu \mathbf{S}) F\|_{P}^{2} .
\end{aligned}
$$

The proof of (20.3) is completed by chosing $\gamma=\gamma(n) \geqslant 2 C(n)$.

Having disposed of the estimate (20.3), we can now make analysis similar to that in the proof of Theorem 8.1 to conclude with inequality (20.2). Here are some details. We consider the approximation $\mu_{m}(x) \rightarrow \mu(x)$, defined by (17.11). The inverse operator $\left(\mathbf{I}-\mu_{m} \mathbf{S}\right)^{-1}$ is bounded in $L^{2 \pm \varepsilon}\left(\mathbb{R}^{n}, d x\right)$ for some positive $\varepsilon=\varepsilon(m)$. By interpolation Lemma 18.2 this operator is also bounded in $L^{P}\left(\mathbb{R}^{n}, d x\right)=L^{2} \log L\left(\mathbb{R}^{n}, d x\right)$, though we have no control of its norm. Now, given any vector field $G \in L^{P}\left(\mathbb{R}^{n}, d \omega\right) \subset L^{P}\left(\mathbb{R}^{n}, d x\right)$ we can solve the equation

$$
\left(\mathbf{I}-\mu_{m} \mathbf{S}\right) F_{m}=G
$$

for $F_{m} \in L^{P}\left(\mathbb{R}^{n}, d x\right)$. Inequality (20.3) remains valid for the operator $\mathbf{I}-\mu_{m} \mathbf{S}$ and provides us with the uniform bound

$$
\begin{aligned}
\left\|F_{m}\right\|_{P} & \leqslant C(n)\|K G\|_{P}+C(n)[K]\left\|F_{m}\right\|_{2} \\
& \leqslant C(n)\|K G\|_{P}+2 C(n)[K]\|K G\|_{2} .
\end{aligned}
$$

The latter step follows from the $L^{2}$-estimate in (17.2). From this point the proof runs as in Theorem 17.1 and, therefore, is omitted.

\section{Extension to the dual space $L^{2} \log ^{-1} L$}

This section is devoted to the following dual version strenghtening of Theorem 20.1.

THEOREM 21.1. - The operator $\boldsymbol{\Pi}=(\mathbf{I}-\mu \mathbf{S})^{-1}$ extends boundedly to the Orlicz spaces.

$$
\Pi: L^{2} \log ^{-1} L\left(\mathbb{R}^{n}, d \omega\right) \rightarrow L^{2} \log ^{-1} L\left(\mathbb{R}^{n}, d x\right)
$$

Moreover

$$
\|\Pi G\|_{L^{2} \log ^{-1} L\left(\mathbb{R}^{n}, d x\right)} \leqslant C(n)[K]\|G\|_{L^{2} \log ^{-1} L\left(\mathbb{R}^{n}, d \omega\right)} .
$$

Proof. - Consider two vector fields $F \in L^{Q}\left(\mathbb{R}^{n}, d x\right)=L^{2} \log ^{-1}\left(\mathbb{R}^{n}, d x\right)$ and $H \in L^{P}\left(\mathbb{R}^{n}, d \omega\right) \subset L^{2} \log L\left(\mathbb{R}^{n}, d x\right)$. As these spaces are dual to each other we can compute the inner product 


$$
\begin{aligned}
\int_{\mathbb{R}^{n}}\langle F, H\rangle & =\int_{\mathbb{R}^{n}}\langle\mathbf{S} R F, \mathbf{S} H\rangle=\int_{\mathbb{R}^{n}}\langle\mathbf{S} F,(\mathbf{I}-\mu \mathbf{S}) \circ \boldsymbol{\Pi} \mathbf{S} H\rangle \\
& =\int_{\mathbb{R}^{n}}\langle(\mathbf{I}-\mathbf{S} \mu) \mathbf{S} F, \boldsymbol{\Pi} \mathbf{S} H\rangle=\int_{\mathbb{R}^{n}}\langle\mathbf{S}(\mathbf{I}-\mu \mathbf{S}) F, \boldsymbol{\Pi} \mathbf{S} H\rangle \\
& =\int_{\mathbb{R}^{n}}\langle(\mathbf{I}-\mu \mathbf{S}) F, \mathbf{S} \boldsymbol{\Pi} \mathbf{S} H\rangle \\
& \leqslant C(n)\|K(\mathbf{I}-\mu \mathbf{S}) F\|_{Q}\left\|K^{-1} \mathbf{S} \Pi \mathbf{S} H\right\|_{P} \\
& \leqslant C(n)\|K(\mathbf{I}-\mu \mathbf{S}) F\|_{Q}\left\|K^{-1} \boldsymbol{\Pi} \mathbf{S} H\right\|_{P} .
\end{aligned}
$$

Here, we have used boundedness of the operator $K^{-1} \mathbf{S} K: L^{P}\left(\mathbb{R}^{n}, d x\right) \rightarrow L^{P}\left(\mathbb{R}^{n}, d x\right)$, which can be verified as follows:

$$
\left\|K^{-1} \mathbf{S} K\right\|_{P}=\left\|K^{-1}(\mathbf{S} K-K \mathbf{S})+\mathbf{S}\right\|_{P} \leqslant\|\mathbf{S} K-K \mathbf{S}\|_{P}+\|\mathbf{S}\|_{P} \leqslant C(n),
$$

by the commutator result (18.16).

However, our focus will be on the operator $K^{-1} \Pi: L^{P}\left(\mathbb{R}^{n}, d \omega\right) \rightarrow L^{P}\left(\mathbb{R}^{n}, d \omega\right)$, for which we need to prove the inequality $\left\|K^{-1} \Pi \Psi\right\|_{P} \leqslant C(n)[K]\|\Psi\|_{P}$, for all $\Psi \in$ $L^{P}\left(\mathbb{R}^{n}, d \omega\right)$. This will give us the desired estimate

$$
\int_{\mathbb{R}^{n}}\langle F, H\rangle \leqslant C(n)[K]\|K(\mathbf{I}-\mu \mathbf{S}) F\|_{Q}\|H\|_{P} .
$$

To this effect, consider an arbitrary $\Phi \in L^{P}\left(\mathbb{R}^{n}, d \omega\right)$ and apply (20.3) to $K^{-1} \Phi$ in place of $F$.

$$
\begin{aligned}
\left\|K^{-1} \Phi\right\|_{P} \leqslant & C(n)\left\|(\mathbf{I}-\mu \mathbf{S}) \Phi+\mu(\mathbf{S} K-K \mathbf{S}) K^{-1} \Phi\right\|_{P}+C(n)[K]\left\|K^{-1} \Phi\right\|_{2} \\
\leqslant & C(n)\|(\mathbf{I}-\mu \mathbf{S}) \Phi\|_{P}+C(n)\|\mathbf{S} K-K \mathbf{S}\|_{P}\left\|K^{-1} \Phi\right\|_{P} \\
& +C(n)[K]\left\|K^{-1} \Phi\right\|_{2} .
\end{aligned}
$$

The term with the commutator is absorbed by the left hand side, because $\|\mathbf{S} K-K \mathbf{S}\|_{P} \leqslant$ $C(n)\|K\|_{\mathrm{BMO}} \leqslant C(n) / \gamma$ and $\gamma$ is sufficiently large.

$$
\left\|K^{-1} \Phi\right\|_{P} \leqslant C(n)\|(\mathbf{I}-\mu \mathbf{S}) \Phi\|_{P}+C(n)[K]\left\|K^{-1} \Phi\right\|_{2} .
$$

To estimate the latter term we make use of Theorem 17.1. Precisely, we use inequality (17.4) with $K^{-1} \Phi$ in place of $F$

$$
\begin{aligned}
\left\|K^{-1} \Phi\right\|_{2} & \leqslant 2\left\|K(\mathbf{I}-\mu \mathbf{S}) K^{-1} \Phi\right\|_{2} \\
& =2\left\|(\mathbf{I}-\mu \mathbf{S}) \Phi+\mu(\mathbf{S} K-K \mathbf{S}) K^{-1} \Phi\right\|_{2} \\
& \leqslant 2\|(\mathbf{I}-\mu \mathbf{S}) \Phi\|_{2}+2\|\mathbf{S} K-K \mathbf{S}\|_{2}\left\|K^{-1} \Phi\right\|_{2} \\
& \leqslant 2\|(\mathbf{I}-\mu \mathbf{S}) \Phi\|_{2}+\frac{C(n)}{\gamma}\left\|K^{-1} \Phi\right\|_{2} .
\end{aligned}
$$

Hence 


$$
\left\|K^{-1} \Phi\right\|_{2} \leqslant 4\|(\mathbf{I}-\mu \mathbf{S}) \Phi\|_{2} .
$$

On substituting this into (21.4), we conclude with the estimate

$$
\begin{aligned}
\left\|K^{-1} \Phi\right\|_{P} & \leqslant C(n)\|(\mathbf{I}-\mu \mathbf{S}) \Phi\|_{P}+4 C(n)[K]\|(\mathbf{I}-\mu \mathbf{S}) \Phi\|_{2} \\
& \leqslant C(n)[K]\|(\mathbf{I}-\mu \mathbf{S}) \Phi\|_{P},
\end{aligned}
$$

which is none other than $\left\|K^{-1} \Pi \Psi\right\|_{P} \leqslant C(n)[K]\|\Psi\|_{P}$, for $\Psi=(\mathbf{I}-\mu \mathbf{S}) \Phi$. As said before, this gives (21.3). Estimate (21.3) holds not only for $H \in L^{P}\left(\mathbb{R}^{n}, d \omega\right)$ but also extends to all $H \in L^{P}\left(\mathbb{R}^{n}, d x\right)$, by an approximation. The final conclusion is that

$$
\|F\|_{Q} \leqslant C(n)[K]\|K(\mathbf{I}-\mu \mathbf{S}) F\|_{Q},
$$

since $L^{Q}\left(\mathbb{R}^{n}, d x\right)$ is the dual space to $L^{P}\left(\mathbb{R}^{n}, d x\right)$. From this, the extendibility of $\Pi$, to a bounded operator on $L^{Q}\left(\mathbb{R}^{n}, d x\right)$ is evident

$$
\begin{aligned}
\|\Pi G\|_{L_{\left(\mathbb{R}^{n}, d x\right)}} & \leqslant C(n)[K]\|K G\|_{L_{\left(\mathbb{R}^{n}, d x\right)}} \\
& \leqslant C(n)[K]\|G\|_{L} Q_{\left(\mathbb{R}^{n}, d \omega\right)}
\end{aligned}
$$

for all $G \in L^{Q}\left(\mathbb{R}^{n}, d \omega\right)$, establishing the theorem.

\section{Higher integrability}

In this section we prove Theorem 4. Incidentally our proof gives also estimates for local solutions of the nonhomogeneous Beltrami equation

$$
\mathcal{F}^{-}(x)=\mu(x) \mathcal{F}^{+}(x)+G(x),
$$

where we assume that $\mathcal{F}$ is a div-curl couple of class $L^{2} \log ^{-1} L\left(\Omega, \mathbb{R}^{n} \times \mathbb{R}^{n}\right)$ and $G$ is a given vector field in $L^{2} \log ^{\alpha} L_{l o c}(\Omega, d \omega)$, with some $\alpha \varepsilon\{-1,0,+1\}$. The theorem is local, thus we can confine ourselves to a cube in $\Omega \subset \mathbb{R}^{n}$. Our objective is to show that $\mathcal{F}$ belongs to $L^{2} \log ^{\alpha} L_{l o c}\left(\Omega, \mathbb{R}^{n} \times \mathbb{R}^{n}\right)$ and

$$
\|\varphi \mathcal{F}\|_{L^{2} \log ^{\alpha} L\left(\mathbb{R}^{n}, d x\right)} \leqslant C\|\varphi G\|_{L^{2} \log ^{\alpha} L\left(\mathbb{R}^{n}, d \omega\right)}+C\||\nabla \varphi||\mathcal{F}|\|_{L^{\frac{2 n}{n+1}\left(\mathbb{R}^{n}, d x\right)}}
$$

for every test function $\varphi \in C_{0}^{\infty}(\Omega)$.

Here the constant $C=C_{\Omega}(n, K)$ depends also on $\Omega$, but not on $\varphi$. We proceed analogously to the proof of Proposition 12.1. Multiplying (22.1) by the test function yields

$$
(\varphi \mathcal{F})^{-}=\mu(\varphi \mathcal{F})^{+}+\varphi G \text { in } \mathbb{R}^{n}
$$

Although $\varphi \mathcal{F}=[\varphi B, \varphi E]$ is no longer a div-curl couple, we see that both $\operatorname{div}, \varphi B=$ $\langle\nabla \varphi \cdot B\rangle$ and curl $\varphi E=\nabla \varphi \star E$ belong to $L^{2} \log ^{-1} L$.

Recall from Section 8 an Orlicz-Sobolev variant of Corollary 8.3. Accordingly, there exists a div-curl couple $\mathcal{F}_{0} \in L^{2} \log ^{-1} L\left(\mathbb{R}^{n}, \mathbb{R}^{n} \times \mathbb{R}^{n}\right)$ for which $\varphi \mathcal{F}-\mathcal{F}_{0}=\Phi$ is in the Orlicz-Sobolev class $\mathcal{W}^{1, Q}\left(\mathbb{R}^{n}, \mathbb{R}^{n} \times \mathbb{R}^{n}\right)$ with $Q(t)=t^{2} \log ^{-1}(\mathrm{e}+t)$. As a matter of fact $\Phi$ is explicitely given by means of the Riesz potentials of $\langle\nabla \varphi \cdot B\rangle$ and $\nabla \varphi \star E$. It is from this observation that we have been able to derive the point-wise inequality (8.16). 
As before, Eq. (22.3) takes the form

$$
(\mathbf{I}-\mu \mathbf{S}) \mathcal{F}_{0}^{-}=\varphi G+\mu \Phi^{+}-\Phi^{-} .
$$

This time, the right hand side is in the space $L^{2} \log ^{\alpha} L\left(\mathbb{R}^{n}, d \omega\right)$, where $d \omega=K^{2} d x$, which is the domain of the inverse operator $\Pi=(\mathbf{I}-\mu \mathbf{S})^{-1}$. Therefore, $\mathcal{F}_{0}^{-}$belongs to $L^{2} \log ^{\alpha} L\left(\mathbb{R}^{n}, d x\right)$ and we have the estimate

$$
\left\|\mathcal{F}_{0}^{-}\right\|_{L^{2} \log ^{\alpha} L\left(\mathbb{R}^{n}, d x\right)} \leqslant C(n, K)\|\varphi G\|_{L^{2} \log ^{\alpha} L\left(\mathbb{R}^{n}, d \omega\right)}+C(n, K)\|\Phi\|_{L^{2} \log ^{\alpha} L\left(\mathbb{R}^{n}, d \omega\right)}
$$

by Theorem 20.1, if $\alpha=1$, Theorem 21.1, if $\alpha=-1$, and Theorem 17.1, if $\alpha=0$. The same holds for $\mathcal{F}^{+}=\mathbf{S} \mathcal{F}_{0}^{-}$, and consequently for $\varphi \mathcal{F}=\mathcal{F}_{0}+\Phi$. Precisely, we have

$$
\|\varphi \mathcal{F}\|_{L^{2} \log ^{\alpha} L\left(\mathbb{R}^{n}, d x\right)} \leqslant C(n, K)\|\varphi G\|_{L^{2} \log ^{\alpha} L\left(\mathbb{R}^{n}, d \omega\right)}+C(n, K)\|\Phi\|_{L^{2} \log ^{\alpha} L\left(\mathbb{R}^{n}, d \omega\right)} .
$$

Letting $G \equiv 0$ and $\alpha=1$ establishes Theorem 4 .

We shall continue this proof to arrive at the uniform bound in (22.2). For this we only need to show that

$$
\|\Phi\|_{L^{2} \log ^{\alpha} L\left(\mathbb{R}^{n}, d \omega\right)} \leqslant C_{\Omega}(n, K)\||\nabla \varphi||\mathcal{F}|\|_{L^{\frac{2 n}{n+1}(\Omega)}} .
$$

For the proof of this inequality, we fix a function $\eta \in C_{0}^{\infty}(3 \Omega)$ which equals 1 on the cube $2 \Omega$. We can write

$$
\begin{aligned}
\|\Phi\|_{L^{2} \log ^{\alpha} L\left(\mathbb{R}^{n}, d \omega\right)} \leqslant & \|\eta \Phi\|_{L^{2} \log ^{\alpha} L(3 \Omega, d \omega)}+\|(1-\eta) \Phi\|_{L^{2} \log ^{\alpha} L\left(\mathbb{R}^{n}-2 \Omega, d \omega\right)} \\
\leqslant & C_{\Omega}(n, K)\|\Phi\|_{L^{\frac{2 n}{n-1}(3 \Omega, d x)}}+C(n)\left\|\frac{\operatorname{diam} \Omega}{\left|x-x_{0}\right|^{n}}\right\|_{L^{2} \log ^{\alpha} L\left(\mathbb{R}^{n}-2 \Omega, d \omega\right)} \\
& \times\||\nabla \varphi||\mathcal{F}|\|_{L^{1}\left(\mathbb{R}^{n}\right)},
\end{aligned}
$$

which is due to the inequality at (8.16). Using Sobolev's inequality the latter terms are dominated by

$$
C_{\Omega}(n, K)\left[\|D \Phi\|_{L^{\frac{2 n}{n+1}\left(\mathbb{R}^{n}, d x\right)}}+\||\nabla \varphi||\mathcal{F}|\|_{L^{1}\left(\mathbb{R}^{n}\right)}\right] .
$$

As said in Section $8, D \Phi$ is expressed by means of the Riesz transforms of $\langle\nabla \varphi, B\rangle$ and $\nabla \varphi \star E$. That is why its norm is controlled by that of $|\nabla \varphi||\mathcal{F}|$. Applying Hölder's inequality to the second term results in (22.6).

\section{Applications to the degenerate elliptic PDEs}

One of the motivations for this study was to solve the Dirichlet problem

$$
\left\{\begin{array}{l}
\operatorname{div} A(x) \nabla u=\operatorname{div} A(x) F \\
\quad u \in \mathcal{H}_{0}^{1, A}(\Omega)
\end{array}\right.
$$

where $A(x)$ is a symmetric matrix with measurable coefficients satisfying the ellipticity bounds

$$
K^{-1}(x)|\xi|^{2} \leqslant\langle A(x) \xi, \xi\rangle \leqslant K(x)|\xi|^{2}
$$


Our only hypothesis concerning the eigenvalues of $A(x)$ is that they are bounded in the following average sense

$$
\int_{\Omega} \mathrm{e}^{\gamma K(x)} d x<\infty
$$

for sufficiently large exponent $\gamma=\gamma(n)$.

The function spaces we shall consider are:

$$
L_{A}^{2}\left(\Omega, \mathbb{R}^{n}\right)=\left\{F ;\|F\|_{A}=\left(\int_{\Omega}|F(x)|_{A}^{2} d x\right)^{1 / 2}<\infty\right\},
$$

where we have used the notation $|\xi|_{A}=\langle A(x) \xi \text {, } \xi\rangle^{1 / 2}$, for $\xi \in \mathbb{R}^{n}$.

$$
\mathcal{H}^{1, A}(\Omega)=\left\{u ;\|\nabla u\|_{A}<\infty\right\} .
$$

Here $\|\nabla u\|_{A}$ is a seminorm which makes $\mathcal{H}^{1, A}(\Omega)$ into a Banach space modulo constant functions. The closure of $C_{0}^{\infty}(\Omega)$ is a Banach space, denoted by $\mathcal{H}_{0}^{1, A}(\Omega)$, which we supply with the norm

$$
\|u\|_{1, A}=\|\nabla u\|_{A}=\left[\int_{\Omega}\langle A(x) \nabla u, \nabla u\rangle d x\right]^{1 / 2} .
$$

Given $F \in L_{A}^{2}\left(\Omega, \mathbb{R}^{n}\right)$, we minimize the variational integral

$$
I[v]=\int_{\Omega}|F-\nabla v|_{A}^{2}
$$

subject to all $v \in \mathcal{H}_{0}^{1, A}(\Omega)$. The minimizer, say $u \in \mathcal{H}_{0}^{1, A}(\Omega)$, is unique and solves the Euler-Lagrange equation (23.1). From this interpretation of the solution the energy estimate (1.36) is immediate. Of course, we assume here that $\|F\|_{A}<\infty$. This is certainly the case if $K F \in L^{2}\left(\Omega, \mathbb{R}^{n}\right)$. As a matter of fact this latter condition implies $L^{2}$-integrability of the gradient of $u$, as in (1.37) for $\Omega$ a cube in $\mathbb{R}^{n}$ or any other regular domain. To see this fact, we first extend equation (23.1) to the double cube $2 \Omega$, like in the proof of Theorem 14.1. The norms in $L^{2} \log ^{\alpha}(2 \Omega)$ for the extended solutions are controlled by those over the original cube $\Omega$. In this way, we look at $u$ as a local solution of (23.1) on the cube $2 \Omega$. Consider the following div-curl couple $\mathcal{F}=[A(x)(\nabla u-F), \nabla u]$. Before making estimates we recall the following general inequality

$$
\|X\|_{L^{2} \log ^{-1} L} \leqslant C_{K}\left\|K^{-1 / 2} X\right\|_{L^{2}}
$$

Hence

$$
\|X\|_{L^{2} \log ^{-1} L}+\|A X\|_{L^{2} \log ^{-1} L} \leqslant C_{K}\|X\|_{L_{A}^{2}}
$$

for all vector fields $X \in L_{A}^{2}$. The constant $C_{K}$ depends only on $\int_{\Omega} \mathrm{e}^{\gamma K}$, details are left to the reader. We see from here that $|\mathcal{F}|$ is in the class $L^{2} \log ^{-1} L(2 \Omega)$. Furthermore, $\mathcal{F}$ 
solves the nonhomogeneous Beltrami equation

$$
\mathcal{F}^{-}(x)=\mu(x) \mathcal{F}^{+}(x)+G(x),
$$

where

$$
\mu(x)=\frac{\mathbf{I}-A(x)}{\mathbf{I}+A(x)}, \quad\|\mu(x)\| \leqslant \frac{K(x)-1}{K(x)+1}
$$

and

$$
G(x)=\left[\frac{-A(x)}{\mathbf{I}+A(x)}\right] F(x), \quad|G(x)| \leqslant|F(x)| .
$$

Now, assume that $K F \in L^{2} \log ^{\alpha}\left(\Omega, \mathbb{R}^{n}\right)$ for some $\alpha=0,1$. The same holds for the extension of $F$ to the double cube. That is, $K F \in L^{2} \log ^{\alpha}\left(2 \Omega, \mathbb{R}^{n}\right)$. It is, therefore, legitimate to apply estimate (22.2) and write

$$
\begin{aligned}
& \|\varphi \nabla u\|_{L^{2} \log ^{\alpha}(2 \Omega)}+\|\varphi A \nabla u\|_{L^{2} \log ^{\alpha}(2 \Omega)} \\
& \quad \leqslant C\|\varphi K F\|_{L^{2} \log ^{\alpha}(2 \Omega)}+C\||\nabla \varphi| K(|F|+|\nabla u|)\|_{L^{\frac{2 n}{n+1}(2 \Omega)}}
\end{aligned}
$$

for every test function $\varphi \in C_{0}^{\infty}(2 \Omega)$. We can certainly take $\varphi \equiv 1$ on $\Omega$. Since the norms over $2 \Omega$ are dominated by the corresponding ones over $\Omega$, we obtain

$$
\begin{aligned}
& \|\nabla u\|_{L^{2} \log ^{\alpha} L(\Omega)}+\|A \nabla u\|_{L^{2} \log ^{\alpha}(\Omega)} \\
& \quad \leqslant C_{\Omega}(n, K)\|K F\|_{L^{2} \log ^{\alpha}(\Omega)}+C_{\Omega}(n, K)\|K \nabla u\|_{L^{\frac{2 n}{n+1}(\Omega)}} .
\end{aligned}
$$

What is left is to estimate the latter term by means of $F$, which can be achieved by using the energy estimate. Indeed, with the aid of Hölder's inequality we find that

$$
\begin{aligned}
\|K \nabla u\|_{L^{\frac{2 n}{n+1}(\Omega)}} & \leqslant C_{K}\left\|K^{-1 / 2} \nabla u\right\|_{L^{2}(\Omega)} \\
& \leqslant C_{K}\|\nabla u\|_{L_{A}^{2}(\Omega)} \leqslant C_{K}\|F\|_{L_{A}^{2}(\Omega)} \\
& \leqslant C_{K}\|K F\|_{L^{2} \log ^{\alpha}(\Omega)} .
\end{aligned}
$$

On substituting this into the previous inequality we conclude this paper with the following Fundamental Estimate for the Dirichlet problem (23.1)

$$
\|\nabla u\|_{L^{2} \log ^{\alpha} L}+\|A \nabla u\|_{L^{2} \log ^{\alpha} L} \leqslant C_{\Omega}(n, K)\|K F\|_{L^{2} \log ^{\alpha} L} .
$$

\section{Acknowledgements}

Research was performed during T. Iwaniec's visit to the Dipartimento di Matematica e Applicazioni “R. Caccioppoli” dell'Università degli Studi di Napoli "Federico II". It was supported by GNAFA-CNR, "Progetto di Ricerca MURST 97012260402" su Equazioni Differenziali e Calcolo delle Variazioni and by NSF Grant DMS-9706611. Both authors are grateful to Zoltan Balogh for his careful reading the first version of the manuscript. 


\section{REFERENCES}

[1] Astala K., Area distortion of quasiconformal mappings, Acta Math. 173 (1994) 37-60.

[2] Astala K., Analytic aspects of quasiconformality, in: Proc. ICM, Vol. 2, 1998, pp. 617-626.

[3] Astala K., Iwaniec T., Koskela P., Martin G., Mappings of BMO-bounded distortion, Mathematische Annalen, to appear.

[4] Astala K., Iwaniec T., Saksman E., The Beltrami operator, Duke Math. J. (1998).

[5] Baernstein A., Montgomery-Smith S.J., Some conjectures about integral means of $\partial f$ and $\bar{\partial} f$, Proc. Mattsfest., to appear.

[6] Bañuelos R., Lindeman A., A martingale study of the Beurling-Ahlfors transform in $\mathbb{R}^{n}$, J. Funct. Anal. 145 (1997) 224-265.

[7] Bers L., Nirenberg L., On linear and nonlinear elliptic boundary value problems in the plane, in: Cremonese (Ed.), Conv. Int.le “Eq. Lineari a Derivate Parziali” (Trieste 1954), Roma, 1955, pp. 141-167.

[8] Boccardo L., Gallouet T., Nonlinear elliptic and parabolic equations involving measure data, J. Funct. Anal. 87 (1989) 149-169.

[9] Bojarski B., Generalized solution of a system of first order differential equations of elliptic type with discontinuous coefficients, Math. Sb. 43 (1957) 451-503.

[10] Brakalova M.A., Jenkins J.A., On solutions of the Beltrami equations, J. d'Analyse Math. 76 (1998) 67-92.

[11] Burkholder D., Sharp inequalities for martingales and stochastic integrals, Asterisque 157/158 (1988) 75-94.

[12] Caccioppoli R., Fondamenti per una teoria generale delle funzioni pseudoanalitiche di una variabile complessa, Rend, Acc. Naz. Lincei 13 (1952) 197-204.

[13] Carozza M., Moscariello G., Passarelli A., Linear elliptic equations with BMO coefficients, Rend. Acc. Naz. Lincei, Ser. IX X (1999) 17-23.

[14] Carozza M., Moscariello G., Passarelli A., Nonlinear equations with growth coefficients in BMO, Rend. Houston J. of Math., to appear.

[15] Chanillo S., Wheeden R.L., Harnack's inequality and mean value inequalities for solutions of degenerate elliptic equations, Comm. PDE 11 (10) (1986) 1111-1134.

[16] Chiarenza F., Frasca M., Longo P., $W^{2, p}$-solvability of the Dirichlet problem for nondivergence elliptic equations with VMO-coefficients, Trans. Amer. Math. Soc. 336 (1993) 841-853.

[17] Coifman R., Lions P.L., Meyer Y., Semmes S., Compensated compactness and Hardy spaces, J. Math. Pures Appl. 72 (1993) 247-286.

[18] Coifman R., Rochberg R., Another characterization of BMO, Proc. AMS 79 (2) (1980) 249-254.

[19] Coifman R., Rochberg R., Weiss G., Factorization theorems for Hardy spaces in several variables, Ann. Math. 103 (1976) 569-645.

[20] Dacorogna B., Direct Methods in the Calculus of Variations, Springer, 1989.

[21] David G., Solutions de l'equation de Beltrami avec $\|\mu\|_{\infty}=1$, Ann. Acad. Sci. Fenn. Ser. A1 Math. 13 (1988) 25-70.

[22] Del Vecchio T., Nonlinear elliptic equations with measure data, Potential Analysis 4 (2) (1995) 185-203.

[23] Di Fazio G., $L^{p}$-estimates for divergence form elliptic equations with discontinuous coefficients, Boll. Un. Mat. It. 7 (1996) 409-420.

[24] De Giorgi E., Unpublished manuscript, 1996. 
[25] Dolzmann G., Hungerbuehler N., Müller S., Uniqueness and maximal regularity for nonlinear elliptic systems of $n$-Laplace type with measure valued right hand side, Preprint, 1998.

[26] Donaldson S.K., Sullivan D.P., Quasiconformal 4-manifolds, Acta Math. 163 (1989) 181252.

[27] Ekeland I., Temam R., Convex Analysis and Variational Problems, North-Holland, 1976.

[28] Eremenko A., Hamilton D., On the area distortion by quasiconformal mappings, Proc. Amer. Math. Soc. 123 (1995) 2793-2797.

[29] Fabes E.B., Kenig C.E., Serapioni R., The local regularity of solutions of degenerate elliptic equations, Comm. Partial Diff. Equations 7 (1982) 77-116.

[30] Finn R., Serrin J., On the Hölder continuity of quasiconformal and elliptic mappings, Trans. Amer. Math. Soc. 89 (1958) 1-15.

[31] Fiorenza A., Sbordone C., Existence and uniqueness results for solutions of nonlinear equations with right hand side in $L^{1}$, Studia Math. 127 (3) (1998) 223-231.

[32] Franchi B., Serapioni R., Serra Cassano F., Irregular solutions of linear degenerate elliptic equations, Potential Analysis 9 (1998) 201-216.

[33] Giannetti F., Verde A., Variational integrals for elliptic complexes, Studia Math. 140 (1) (2000) 79-98.

[34] Greco L., Iwaniec T., Moscariello G., Limits of the improved integrability of the volume forms, Indiana Univ. Math. J. 44 (2) (1995) 305-339.

[35] Greco L., Iwaniec T., Sbordone C., Inverting the p-harmonic operator, Manuscripta Math. 92 (1997) 249-258.

[36] Gustavsson J., Peetre J., Interpolation of Orlicz spaces, Studia Math. LX (1977) 33-59.

[37] Heinonen J., Koskela P., Sobolev mappings with integrable dilatation, Arch. Rat. Mech. Anal. 125 (1993) 81-97.

[38] Iwaniec T., Nonlinear Cauchy-Riemann operators in $\mathbb{R}^{n}$, Trans. AMS, to appear.

[39] Iwaniec T., Koskela P., Martin G., Mappings of BMO-bounded distorsion and Beltrami type operators, Preprint Univ. of Jyväskyla, 1998.

[40] Iwaniec T., Lutoborski A., Integral estimates for null Lagrangians, Arch. Rat. Mech. Anal. 125 (1993) 25-79.

[41] Iwaniec T., Martin G., Quasiregular mappings in even dimension, Acta Math. 170 (1993) 29-81.

[42] Iwaniec T., Martin G., Riesz Transforms and related singular integrals, J. Reine Angew. Math. 473 (1996) 25-57.

[43] Iwaniec T., Sbordone C., On the integrability of the Jacobian under minimal hypotheses, Arch. Rat. Mech. Anal. 119 (1992) 129-143.

[44] Iwaniec T., Sbordone C., Riesz transforms and elliptic pde's with VMO coefficients, J. d'Analyse Math. 74 (1998) 183-212.

[45] Iwaniec T., Sbordone C., Div-curl fields of finite distorsion, C.R. Acad. Sci. Paris, Serie I 327 (1998) 729-734.

[46] Iwaniec T., Scott C., Stroffolini B., Nonlinear Hodge theory on manifolds with boundary, Annali di Matematica pura ed applicata (IV) CLXXV (1999) 37-115.

[47] Iwaniec T., Šverák V., On mappings with integrable dilatation, Proc. Amer. Math. Soc. 118 (1993) 181-188.

[48] Iwaniec T., Verde A., A study of Jacobians in Hardy-Orlicz spaces, Proc. Roy. Soc. Edinburgh 29A (1999) 539-570.

[49] John O., Malý J., Stará J., Nowhere continuous solutions to elliptic systems, Comm. Math. Univ. Carolinae 30 (1) (1989) 33-43. 
[50] Kilpeläinen T., Malý J., Degenerate elliptic equations with measure data and nonlinear potentials, Ann. Scuola Norm. Sup. Pisa, Ser. IV 19 (1992) 591-613.

[51] Koskela P., Manfredi J., Villamore E., Regularity theory and traces of As-harmonic functions, Trans. Amer. Math. Soc. 348 (2) (1996) 755-766.

[52] Krasnosel'skii M.A., Rutickii Yu.B., Convex Functions and Orlicz spaces, P. Noordhoff Ltd., Groningen, 1961, translation.

[53] Leonetti F., Nesi V., Quasiconformal solutions to certain first order systems and the proof of a conjecture of G.W. Milton, J. Math. Pures Appl. 76 (1997) 1-16.

[54] Manfredi J., Villamor E., Mappings with integrable dilatation in higher dimensions, Bull. Amer. Math. Soc. 32 (1995) 235-240.

[55] Meyers N., An $L^{p}$-estimate for the gradient of solutions of second order elliptic divergence equations, Ann. Sc. Norm. Sup. Pisa 17 (1963) 189-206.

[56] Migliaccio L., Moscariello G., Higher integrability of Div-Curl Products (1997), Ricerche di Matematica, to appear.

[57] Iwaniec T., Migliaccio L., Moscariello G., Passarelli di Napoli A., Integrability Theory of Nonlinear Elliptic Complexes, to appear.

[58] Modica G., Quasiminimi di alcuni funzionali degeneri, Annali di Matematica (1984) 121143.

[59] Moscariello G., On the integrability of the Jacobian in Orlicz spaces, Math. Japonica 40 (2) (1994) 323-329.

[60] Müller S., Higher integrability of determinants and weak convergence in $L^{1}$, J. Reine Angew. Math. 412 (1990) 20-34.

[61] Murat F., Equations non linéaires avec second membre $L^{1}$ on mésure, Preprint, 1994.

[62] Palagachev D.K., Quasilinear elliptic equations with VMO coefficients, Trans. Amer. Math. Soc. 347 (1995) 2481-2493.

[63] Rao M.M., Ren Z.D., Theory of Orlicz Spaces, Marcel Dekker, New York, 1991.

[64] Ryazanov V., Srebro U., Yakubov E., BMO-quasiconformal mappings, Preprint 155, University of Helsinki, August 1997.

[65] Sbordone C., New estimates for div-curl products and very weak solutions of pde's, Ann. Scuola Norm. Sup. Pisa IV XXV (4) (1997) 739-756.

[66] Serrin J., Pathological solutions of elliptic differential equations, Ann. Sc. Norm. Sup. Pisa 18 (1964) 385-387.

[67] Souček J., Singular solutions to linear elliptic systems, Comm. Math. Univ. Carolinae 25 (1984) 273-281.

[68] Stampacchia G., Le probléme de Dirichlet pour les equations elliptiques du second ordre a coefficients discontinus, Ann. Inst. Fourier, Grenoble 15 (1965) 189-258.

[69] Stein E., Singular Integrals and Differentiability Properties of Functions, Princeton University Press, 1970.

[70] Stein E., Harmonic Analysis, Princeton University Press, 1993.

[71] Šverák V., Rank-one convexity does not imply quasiconvexity, Proc. Royal Soc. Edinburgh 120A (1992) 185-189.

[72] Trudinger N., On the regularity of generalized solutions of linear nonuniformly elliptic equations, Arch. Rat. Mech. Anal. 42 (1971) 51-62.

[73] Tukia P., Compactness properties of $\mu$-homeomorphisms, Ann. Acad. Sci., Ser. A1 Math. 16 (1991) 47-69.

[74] Vekua I.N., Generalized Analytic Functions, Pure and Applied Mathematics, Vol. 25, Pergamon Press, Oxford. 